\title{
Assessing the current state of AMBER force field modifications for DNA.
}

\section{Supporting Information.}

Table S1 Average structural parameters for the DDD system with the $\varepsilon / \zeta_{O L 1}{ }^{+} X_{O L 4}$ force field modification.

\begin{tabular}{|c|c|c|c|c|c|c|c|c|}
\hline & & & & & \multicolumn{2}{|c|}{ TIP3P } & \multicolumn{2}{|c|}{ OPC } \\
\hline & & & & & \multicolumn{2}{|c|}{ ez1ol4 } & \multicolumn{2}{|c|}{ ez1ol4 } \\
\hline & NMR Avg & NMR Std Dev & X-ray Avg & X-ray Std Dev & Avg & Std Dev & Avg & Std Dev \\
\hline shear & 0.00 & 0.24 & 0.06 & 0.21 & 0.00 & 0.10 & 0.00 & 0.10 \\
\hline stretch & -0.34 & 0.09 & -0.23 & 0.11 & -0.02 & 0.04 & -0.03 & 0.04 \\
\hline stagger & -0.12 & 0.11 & 0.06 & 0.15 & -0.01 & 0.16 & 0.02 & 0.15 \\
\hline buckle & -0.02 & 2.91 & 0.24 & 5.93 & 0.03 & 4.70 & 0.01 & 4.40 \\
\hline propeller & -17.58 & 3.93 & -13.34 & 5.56 & -12.40 & 2.68 & -12.14 & 2.57 \\
\hline opening & -1.10 & 1.21 & 1.31 & 3.41 & 0.21 & 1.76 & 0.18 & 1.66 \\
\hline shift & 0.00 & 0.32 & 0.03 & 0.26 & 0.00 & 0.19 & 0.00 & 0.18 \\
\hline tilt & 0.01 & 1.09 & 0.30 & 2.49 & 0.00 & 1.26 & 0.00 & 1.22 \\
\hline slide & -0.21 & 0.18 & 0.07 & 0.49 & -0.09 & 0.25 & -0.17 & 0.24 \\
\hline rise & 3.20 & 0.16 & 3.29 & 0.11 & 3.32 & 0.06 & 3.31 & 0.06 \\
\hline roll & 3.03 & 3.54 & 1.98 & 3.16 & 3.26 & 1.97 & 2.42 & 1.99 \\
\hline twist & 35.71 & 1.91 & 34.22 & 5.23 & 35.31 & 1.27 & 35.68 & 1.27 \\
\hline xdisp & -0.81 & 0.35 & -0.23 & 0.49 & -0.73 & 0.52 & -0.73 & 0.51 \\
\hline ydisp & 0.00 & 0.45 & 0.05 & 0.92 & 0.00 & 0.27 & 0.00 & 0.25 \\
\hline helical-rise & 3.18 & 0.12 & 3.29 & 0.11 & 3.28 & 0.10 & 3.27 & 0.09 \\
\hline helical-inclination & 5.04 & 5.93 & 4.02 & 6.70 & 5.51 & 3.33 & 4.07 & 3.35 \\
\hline tip & -0.01 & 1.87 & -0.75 & 4.70 & 0.00 & 2.11 & 0.00 & 2.03 \\
\hline Helical-twist & 36.03 & 1.71 & 34.56 & 4.83 & 36.31 & 1.22 & 36.58 & 1.22 \\
\hline major width & 19.56 & 0.22 & 19.12 & 0.49 & 19.44 & 0.26 & 19.49 & 0.25 \\
\hline minor width & 12.22 & 0.25 & 12.19 & 0.26 & 12.30 & 0.15 & 12.30 & 0.14 \\
\hline pucker & 137.10 & 14.22 & 129.50 & 26.73 & 148.13 & 6.38 & 147.86 & 6.09 \\
\hline alpha & 298.80 & 6.19 & 299.20 & 6.64 & 290.10 & 3.93 & 291.13 & 3.86 \\
\hline beta & 172.40 & 4.90 & 175.70 & 10.44 & 170.56 & 3.94 & 171.85 & 3.99 \\
\hline gamma & 50.28 & 4.92 & 56.52 & 7.33 & 54.35 & 3.67 & 54.13 & 3.56 \\
\hline delta & 126.70 & 9.00 & 122.80 & 19.58 & 131.02 & 3.73 & 130.67 & 3.62 \\
\hline epsilon & 188.50 & 5.16 & 190.40 & 27.36 & 194.46 & 6.66 & 191.28 & 6.69 \\
\hline zeta & 257.10 & 11.96 & 251.30 & 37.64 & 249.35 & 6.89 & 251.40 & 6.75 \\
\hline chi & 249.20 & 7.85 & 243.90 & 14.66 & 250.39 & 3.88 & 249.91 & 3.74 \\
\hline
\end{tabular}


Table S2. Average structural parameters for the 1FZX systems.

\begin{tabular}{|c|c|c|c|c|c|c|c|c|c|c|c|c|c|c|c|c|c|c|}
\hline & & \multirow[b]{3}{*}{$\begin{array}{c}\text { NMR Std } \\
\text { Dev }\end{array}$} & \multicolumn{8}{|c|}{ Tip3p } & \multicolumn{8}{|c|}{ opc } \\
\hline & \multirow[b]{2}{*}{$\begin{array}{l}\text { NMR } \\
\text { Avg }\end{array}$} & & \multicolumn{2}{|c|}{ Bsc0 } & \multicolumn{2}{|c|}{$\varepsilon / \zeta_{O L 1}+X_{O L 4}$} & \multicolumn{2}{|c|}{ ol15 } & \multicolumn{2}{|c|}{ bsc1 } & \multicolumn{2}{|c|}{$\mathrm{Bsc0}$} & \multicolumn{2}{|c|}{$\varepsilon / \zeta_{O L 1}+X_{O L 4}$} & \multicolumn{2}{|c|}{ ol15 } & \multicolumn{2}{|c|}{ bsc1 } \\
\hline & & & Avg & $\begin{array}{l}\text { Std } \\
\text { Dev }\end{array}$ & Avg & $\begin{array}{l}\text { Std } \\
\text { Dev }\end{array}$ & Avg & $\begin{array}{l}\text { Std } \\
\text { Dev }\end{array}$ & Avg & $\begin{array}{l}\text { Std } \\
\text { Dev }\end{array}$ & Avg & $\begin{array}{l}\text { Std } \\
\text { Dev }\end{array}$ & Avg & $\begin{array}{l}\text { Std } \\
\text { Dev }\end{array}$ & Avg & $\begin{array}{l}\text { Std } \\
\text { Dev }\end{array}$ & Avg & $\begin{array}{l}\text { Std } \\
\text { Dev }\end{array}$ \\
\hline shear & 0.17 & 0.18 & 0.10 & 0.10 & 0.11 & 0.10 & 0.11 & 0.10 & 0.11 & 0.10 & 0.10 & 0.10 & 0.11 & 0.10 & 0.11 & 0.10 & 0.11 & 0.10 \\
\hline stretch & -0.30 & 0.07 & -0.01 & 0.04 & -0.01 & 0.04 & -0.01 & 0.04 & -0.01 & 0.04 & -0.01 & 0.04 & -0.02 & 0.04 & -0.02 & 0.04 & -0.01 & 0.04 \\
\hline stagger & 0.34 & 0.26 & -0.04 & 0.18 & -0.06 & 0.17 & -0.07 & 0.17 & -0.03 & 0.17 & 0.03 & 0.17 & -0.01 & 0.17 & -0.02 & 0.17 & 0.01 & 0.17 \\
\hline buckle & 0.47 & 8.30 & 3.88 & 5.36 & 6.01 & 5.41 & 6.39 & 5.37 & 4.25 & 4.98 & 2.20 & 4.83 & 3.44 & 4.86 & 3.72 & 4.88 & 3.14 & 4.58 \\
\hline propeller & -13.94 & 5.50 & $\begin{array}{c}- \\
14.29 \\
\end{array}$ & 3.37 & $\begin{array}{c}- \\
13.88\end{array}$ & 2.88 & $\begin{array}{c}- \\
13.56 \\
\end{array}$ & 2.91 & $\begin{array}{c}- \\
10.98 \\
\end{array}$ & 3.05 & $\begin{array}{c}- \\
14.60 \\
\end{array}$ & 3.09 & $\begin{array}{c}- \\
14.14\end{array}$ & 2.75 & $\begin{array}{c}- \\
13.58 \\
\end{array}$ & 2.77 & $\begin{array}{c}- \\
11.21 \\
\end{array}$ & 2.90 \\
\hline opening & 0.17 & 3.86 & 0.95 & 2.02 & 1.18 & 1.99 & 1.20 & 1.99 & 0.42 & 1.91 & 0.56 & 1.90 & 0.78 & 1.87 & 0.82 & 1.87 & 0.11 & 1.82 \\
\hline shift & -0.11 & 0.32 & -0.11 & 0.16 & -0.18 & 0.17 & -0.18 & 0.17 & -0.16 & 0.18 & -0.10 & 0.15 & -0.14 & 0.16 & -0.17 & 0.16 & -0.14 & 0.17 \\
\hline tilt & -1.75 & 1.83 & -0.60 & 1.15 & -0.88 & 1.14 & -0.94 & 1.13 & -1.11 & 1.14 & -0.54 & 1.11 & -0.73 & 1.10 & -0.87 & 1.08 & -1.00 & 1.10 \\
\hline slide & -0.89 & 0.15 & -0.55 & 0.28 & -0.29 & 0.26 & -0.23 & 0.26 & -0.50 & 0.25 & -0.57 & 0.25 & -0.38 & 0.24 & -0.33 & 0.24 & -0.55 & 0.23 \\
\hline rise & 3.06 & 0.09 & 3.34 & 0.08 & 3.32 & 0.07 & 3.32 & 0.07 & 3.32 & 0.07 & 3.32 & 0.07 & 3.31 & 0.07 & 3.31 & 0.07 & 3.30 & 0.07 \\
\hline roll & 0.39 & 3.60 & 2.55 & 2.36 & 3.08 & 2.24 & 2.90 & 2.25 & 1.91 & 2.11 & 1.34 & 2.22 & 1.42 & 2.15 & 1.52 & 2.15 & 1.21 & 1.96 \\
\hline twist & 36.23 & 1.97 & 32.80 & 1.47 & 34.23 & 1.35 & 34.16 & 1.38 & 33.85 & 1.34 & 33.55 & 1.42 & 34.72 & 1.29 & 34.54 & 1.35 & 34.14 & 1.30 \\
\hline xdisp & -1.48 & 0.47 & -1.54 & 0.57 & -1.10 & 0.50 & -0.97 & 0.53 & -1.29 & 0.53 & -1.35 & 0.52 & -0.97 & 0.48 & -0.92 & 0.51 & -1.26 & 0.49 \\
\hline ydisp & -0.06 & 0.31 & 0.11 & 0.31 & 0.15 & 0.28 & 0.16 & 0.29 & 0.10 & 0.31 & 0.11 & 0.29 & 0.13 & 0.26 & 0.16 & 0.28 & 0.09 & 0.29 \\
\hline helical-rise & 3.05 & 0.19 & 3.20 & 0.13 & 3.23 & 0.11 & 3.23 & 0.11 & 3.22 & 0.11 & 3.22 & 0.12 & 3.24 & 0.10 & 3.23 & 0.10 & 3.22 & 0.11 \\
\hline $\begin{array}{c}\text { helical- } \\
\text { inclination }\end{array}$ & 0.76 & 6.13 & 5.05 & 4.41 & 5.55 & 3.94 & 5.40 & 4.00 & 3.78 & 3.79 & 2.91 & 4.14 & 2.74 & 3.76 & 3.09 & 3.84 & 2.58 & 3.54 \\
\hline tip & 2.71 & 2.82 & 0.81 & 2.18 & 1.31 & 2.03 & 1.39 & 2.06 & 1.66 & 2.09 & 0.69 & 2.07 & 1.06 & 1.93 & 1.25 & 1.94 & 1.47 & 1.99 \\
\hline Helical-twist & 36.50 & 1.79 & 33.89 & 1.35 & 35.19 & 1.24 & 35.14 & 1.27 & 34.78 & 1.27 & 34.48 & 1.30 & 35.52 & 1.20 & 35.38 & 1.24 & 34.98 & 1.22 \\
\hline major width & 19.41 & 0.11 & 20.03 & 0.33 & 19.64 & 0.28 & 19.55 & 0.27 & 19.73 & 0.28 & 20.05 & 0.29 & 19.71 & 0.26 & 19.62 & 0.25 & 19.78 & 0.27 \\
\hline minor width & 12.37 & 0.24 & 12.55 & 0.21 & 12.30 & 0.18 & 12.25 & 0.18 & 12.39 & 0.17 & 12.56 & 0.19 & 12.34 & 0.17 & 12.29 & 0.16 & 12.40 & 0.17 \\
\hline pucker & 130.5 & 21.31 & 127.1 & 7.80 & 143.0 & 6.48 & 144.0 & 6.31 & 145.6 & 7.33 & 129.1 & 6.93 & 143.1 & 6.05 & 144.3 & 5.95 & 146.4 & 6.46 \\
\hline alpha & 290.1 & 5.79 & 288.0 & 6.07 & 290.9 & 4.02 & 290.2 & 4.50 & 286.4 & 5.87 & 288.6 & 6.38 & 292.2 & 3.96 & 291.3 & 4.39 & 287.2 & 5.95 \\
\hline beta & 183.1 & 9.26 & 169.1 & 3.87 & 172.3 & 3.74 & 170.1 & 5.01 & 166.7 & 6.05 & 169.4 & 4.16 & 173.8 & 3.82 & 172.4 & 5.12 & 167.6 & 6.00 \\
\hline gamma & 58.2 & 4.37 & 59.0 & 4.87 & 55.2 & 3.65 & 54.9 & 3.64 & 60.2 & 7.09 & 59.2 & 5.14 & 54.9 & 3.58 & 54.5 & 3.53 & 59.9 & 7.00 \\
\hline delta & 132.5 & 12.15 & 118.8 & 4.31 & 127.7 & 3.78 & 128.7 & 3.61 & 131.8 & 4.10 & 119.7 & 3.99 & 127.3 & 3.61 & 128.3 & 3.45 & 132.0 & 3.73 \\
\hline epsilon & 173.0 & 6.20 & 193.9 & 7.10 & 190.2 & 6.61 & 194.5 & 7.78 & 197.4 & 5.81 & 191.6 & 6.96 & 186.0 & 6.58 & 189.4 & 7.79 & 194.9 & 5.75 \\
\hline zeta & 261.5 & 9.12 & 259.6 & 8.07 & 254.8 & 6.90 & 250.1 & 7.79 & 250.2 & 7.41 & 260.3 & 7.67 & 257.7 & 6.71 & 254.0 & 7.59 & 251.5 & 7.15 \\
\hline chi & 238.2 & 9.19 & 239.5 & 5.17 & 248.0 & 4.01 & 249.3 & 3.96 & 245.2 & 4.14 & 239.7 & 4.72 & 247.4 & 3.78 & 248.7 & 3.73 & 245.1 & 3.84 \\
\hline
\end{tabular}


Table S3. Average structural parameters for the 110T systems.

\begin{tabular}{|c|c|c|c|c|c|c|c|c|c|c|c|c|c|c|c|c|c|c|}
\hline & & & \multicolumn{8}{|c|}{ Tip3p } & \multicolumn{8}{|c|}{ opc } \\
\hline & & & \multicolumn{2}{|c|}{$\mathrm{Bsc0}$} & \multicolumn{2}{|c|}{$\varepsilon / \zeta_{O L 1}+X_{O L 4}$} & \multicolumn{2}{|c|}{ ol15 } & \multicolumn{2}{|c|}{ bsc1 } & \multicolumn{2}{|c|}{$\mathrm{Bsc0}$} & \multicolumn{2}{|c|}{$\varepsilon / \zeta_{O L 1}+X_{O L 4}$} & \multicolumn{2}{|c|}{ ol15 } & \multicolumn{2}{|c|}{ bsc1 } \\
\hline & $\begin{array}{l}\text { Xray } \\
\text { Avg }\end{array}$ & $\begin{array}{c}\text { Xray } \\
\text { Std Dev }\end{array}$ & Avg & $\begin{array}{l}\text { Std } \\
\text { Dev }\end{array}$ & Avg & $\begin{array}{l}\text { Std } \\
\text { Dev }\end{array}$ & Avg & $\begin{array}{l}\text { Std } \\
\text { Dev }\end{array}$ & Avg & Std Dev & Avg & $\begin{array}{l}\text { Std } \\
\text { Dev }\end{array}$ & Avg & $\begin{array}{l}\text { Std } \\
\text { Dev }\end{array}$ & Avg & $\begin{array}{l}\text { Std } \\
\text { Dev }\end{array}$ & Avg & Std Dev \\
\hline shear & -0.02 & 0.26 & 0.00 & 0.16 & -0.01 & 0.17 & 0.00 & 0.16 & 0.01 & 0.16 & 0.00 & 0.16 & 0.00 & 0.16 & 0.00 & 0.16 & 0.00 & 0.15 \\
\hline stretch & -0.17 & 0.01 & -0.08 & 0.05 & -0.08 & 0.08 & -0.08 & 0.06 & -0.11 & 0.30 & -0.09 & 0.05 & -0.09 & 0.05 & -0.09 & 0.05 & -0.08 & 0.05 \\
\hline stagger & -0.04 & 0.06 & -0.11 & 0.18 & -0.01 & 0.21 & -0.02 & 0.18 & -0.17 & 0.38 & -0.08 & 0.18 & 0.00 & 0.18 & 0.00 & 0.18 & -0.17 & 0.16 \\
\hline buckle & -0.14 & 4.19 & -0.02 & 4.73 & -0.01 & 4.86 & -0.03 & 4.61 & -0.02 & 4.84 & 0.05 & 4.50 & -0.02 & 4.56 & 0.02 & 4.52 & -0.04 & 4.42 \\
\hline propeller & 1.41 & 5.49 & 0.84 & 4.21 & -1.16 & 5.67 & 0.17 & 4.35 & -1.00 & 11.70 & -0.22 & 4.00 & -1.60 & 4.26 & -0.79 & 4.29 & -1.95 & 3.61 \\
\hline opening & -2.02 & 0.64 & -0.45 & 1.58 & -0.27 & 2.62 & -0.26 & 1.65 & -0.93 & 7.14 & -0.69 & 1.54 & -0.45 & 1.61 & -0.46 & 1.61 & -0.39 & 1.46 \\
\hline shift & 0.16 & 0.59 & 0.00 & 0.22 & 0.02 & 0.27 & 0.01 & 0.21 & -0.01 & 0.30 & 0.00 & 0.21 & 0.00 & 0.21 & -0.01 & 0.20 & 0.00 & 0.18 \\
\hline tilt & -0.12 & 1.24 & -0.01 & 1.83 & 0.09 & 2.13 & 0.06 & 1.80 & 0.19 & 4.57 & 0.03 & 1.77 & 0.01 & 1.79 & 0.02 & 1.77 & -0.10 & 1.65 \\
\hline slide & 1.41 & 2.97 & 0.84 & 0.32 & 1.10 & 0.36 & 1.08 & 0.33 & 1.48 & 0.33 & 0.80 & 0.29 & 0.94 & 0.32 & 0.97 & 0.31 & 1.42 & 0.27 \\
\hline rise & -3.36 & 0.18 & -3.47 & 0.13 & -3.42 & 0.15 & -3.42 & 0.13 & -3.41 & 0.28 & -3.44 & 0.12 & -3.41 & 0.12 & -3.40 & 0.12 & -3.44 & 0.12 \\
\hline roll & -2.13 & 3.33 & -2.33 & 2.63 & -3.39 & 3.96 & -3.94 & 2.41 & 0.42 & 9.42 & -2.86 & 2.43 & -4.40 & 2.52 & -4.72 & 2.44 & -1.29 & 2.47 \\
\hline twist & 37.78 & 21.69 & 34.06 & 2.34 & 31.57 & 3.38 & 31.84 & 2.70 & 32.11 & 6.88 & 33.85 & 2.00 & 32.17 & 2.44 & 32.09 & 2.36 & 32.08 & 2.60 \\
\hline xdisp & 14.94 & 22.52 & 6.23 & 3.43 & 4.88 & 3.18 & 4.92 & 2.85 & 5.72 & 2.39 & 6.39 & 3.63 & 4.19 & 3.05 & 4.34 & 2.78 & 5.36 & 2.05 \\
\hline ydisp & -2.85 & 4.09 & 0.03 & 2.22 & 0.02 & 2.10 & 0.00 & 1.82 & -0.05 & 1.51 & 0.00 & 2.47 & 0.00 & 2.01 & -0.01 & 1.83 & 0.03 & 1.24 \\
\hline helical-rise & -2.99 & 0.29 & -3.07 & 0.69 & -3.42 & 0.60 & -3.36 & 0.58 & -3.08 & 0.58 & -3.19 & 0.67 & -3.52 & 0.53 & -3.44 & 0.54 & -3.21 & 0.49 \\
\hline helical-inclination & 0.10 & 6.47 & -2.47 & 7.58 & -7.58 & 8.14 & -7.41 & 7.13 & 0.44 & 10.31 & -4.33 & 7.60 & -9.68 & 7.21 & -9.39 & 7.04 & -1.51 & 6.93 \\
\hline tip & -3.41 & 6.34 & 0.09 & 6.29 & 0.02 & 5.88 & -0.02 & 5.33 & -0.14 & 5.33 & -0.04 & 6.43 & -0.02 & 5.53 & -0.08 & 5.20 & 0.19 & 4.43 \\
\hline Helical-twist & 38.00 & 21.68 & 35.14 & 2.39 & 32.80 & 3.94 & 32.90 & 2.63 & 33.46 & 10.68 & 34.86 & 1.99 & 33.34 & 2.30 & 33.23 & 2.26 & 32.87 & 2.41 \\
\hline major width & 14.80 & 0.18 & 15.05 & 0.37 & 14.99 & 0.65 & 15.07 & 0.40 & 15.79 & 0.54 & 14.95 & 0.34 & 15.02 & 0.37 & 15.03 & 0.38 & 15.82 & 0.30 \\
\hline minor width & 11.76 & 0.14 & 11.97 & 0.16 & 12.03 & 0.43 & 12.05 & 0.15 & 12.80 & 0.39 & 11.92 & 0.15 & 12.03 & 0.15 & 12.02 & 0.14 & 12.84 & 0.14 \\
\hline pucker & 93.3 & 61.3 & 109.6 & 25.45 & 108.1 & 19.74 & 102.5 & 15.76 & 152.0 & 7.53 & 109.4 & 26.40 & 107.2 & 22.23 & 101.4 & 17.67 & 153.6 & 6.33 \\
\hline alpha & 130.8 & 64.5 & 153.6 & 15.14 & 166.1 & 20.34 & 158.6 & 19.54 & 202.1 & 10.31 & 147.4 & 12.80 & 153.2 & 17.60 & 150.6 & 17.88 & 201.8 & 10.44 \\
\hline beta & 194.1 & 24.2 & 185.7 & 7.70 & 190.3 & 8.13 & 201.4 & 7.38 & 181.6 & 6.35 & 185.0 & 7.34 & 191.4 & 8.02 & 202.5 & 7.22 & 180.8 & 6.15 \\
\hline gamma & 116.1 & 63.0 & 106.3 & 12.92 & 92.0 & 16.29 & 94.0 & 15.33 & 62.5 & 13.79 & 111.4 & 10.17 & 102.5 & 14.25 & 101.9 & 14.06 & 61.1 & 12.14 \\
\hline delta & 118.9 & 23.5 & 116.2 & 3.71 & 113.8 & 4.92 & 111.5 & 4.14 & 138.5 & 4.19 & 116.3 & 3.38 & 113.5 & 4.04 & 111.9 & 3.79 & 139.8 & 4.01 \\
\hline epsilon & 247.6 & 24.6 & 256.9 & 11.44 & 244.3 & 11.94 & 243.1 & 11.07 & 225.4 & 5.57 & 256.6 & 12.16 & 248.0 & 10.24 & 246.4 & 9.02 & 225.6 & 4.27 \\
\hline zeta & 157.6 & 114.7 & 170.8 & 23.74 & 171.0 & 24.97 & 177.1 & 19.04 & 231.6 & 14.23 & 163.4 & 25.01 & 162.6 & 25.16 & 174.4 & 17.23 & 234.4 & 11.68 \\
\hline chi & 131.1 & 72.1 & 132.0 & 4.68 & 133.3 & 7.07 & 132.2 & 4.00 & 159.9 & 5.38 & 131.0 & 3.97 & 131.1 & 3.87 & 131.4 & 3.70 & 160.4 & 4.89 \\
\hline
\end{tabular}


Table S4. Average structural parameters for the 1 SK5 systems.

\begin{tabular}{|c|c|c|c|c|c|c|c|c|c|c|c|c|c|c|c|c|c|c|}
\hline & & & \multicolumn{8}{|c|}{ Tip3p } & \multicolumn{8}{|c|}{ OPC } \\
\hline & & & \multicolumn{2}{|c|}{ Bsc0 } & \multicolumn{2}{|c|}{$\varepsilon / \zeta_{O L 1}+X$ OL4 } & \multicolumn{2}{|c|}{ ol15 } & \multicolumn{2}{|c|}{ bsc1 } & \multicolumn{2}{|c|}{ Bsc0 } & \multicolumn{2}{|c|}{$\varepsilon / \zeta_{\mathrm{OL} 1}+X_{\mathrm{OL} 4}$} & \multicolumn{2}{|c|}{ ol15 } & \multicolumn{2}{|c|}{ bsc1 } \\
\hline & $\begin{array}{l}\text { Xray } \\
\text { Avg }\end{array}$ & $\begin{array}{c}\text { Xray Std } \\
\text { Dev }\end{array}$ & Avg & $\begin{array}{l}\text { Std } \\
\text { Dev }\end{array}$ & Avg & $\begin{array}{l}\text { Std } \\
\text { Dev }\end{array}$ & Avg & $\begin{array}{l}\text { Std } \\
\text { Dev }\end{array}$ & Avg & $\begin{array}{l}\text { Std } \\
\text { Dev }\end{array}$ & Avg & $\begin{array}{l}\text { Std } \\
\text { Dev }\end{array}$ & Avg & $\begin{array}{l}\text { Std } \\
\text { Dev }\end{array}$ & Avg & $\begin{array}{l}\text { Std } \\
\text { Dev }\end{array}$ & Avg & $\begin{array}{l}\text { Std } \\
\text { Dev }\end{array}$ \\
\hline shear & 0.01 & 0.07 & -0.01 & 0.13 & 0.00 & 0.10 & 0.00 & 0.11 & 0.00 & 0.10 & 0.00 & 0.10 & 0.00 & 0.10 & 0.00 & 0.10 & 0.00 & 0.10 \\
\hline stretch & -0.12 & 0.02 & -0.02 & 0.13 & 0.00 & 0.05 & 0.00 & 0.07 & -0.03 & 0.12 & -0.01 & 0.08 & 0.00 & 0.04 & 0.00 & 0.05 & 0.00 & 0.06 \\
\hline stagger & -0.02 & 0.10 & -0.04 & 0.27 & -0.07 & 0.18 & -0.05 & 0.21 & 0.06 & 0.31 & 0.00 & 0.27 & -0.03 & 0.18 & -0.02 & 0.18 & 0.03 & 0.18 \\
\hline buckle & -2.49 & 4.87 & 0.40 & 6.60 & 0.35 & 5.65 & -0.55 & 6.76 & -0.01 & 4.95 & -0.49 & 5.46 & -0.14 & 5.31 & -0.13 & 5.40 & -0.18 & 4.87 \\
\hline propeller & -15.86 & 3.84 & 13.47 & 6.01 & 15.36 & 3.18 & 14.35 & 4.66 & $\begin{array}{c}- \\
11.48 \\
\end{array}$ & 4.59 & 13.32 & 5.59 & $\begin{array}{c}- \\
15.55 \\
\end{array}$ & 3.12 & 15.58 & 2.95 & $\begin{array}{c}- \\
12.78 \\
\end{array}$ & 3.32 \\
\hline opening & 2.20 & 1.07 & 2.47 & 3.72 & 2.06 & 2.27 & 1.97 & 3.01 & 1.88 & 3.33 & 1.91 & 2.81 & 1.82 & 2.18 & 1.90 & 2.23 & 0.95 & 2.23 \\
\hline shift & -0.06 & 0.15 & 0.03 & 0.42 & 0.00 & 0.16 & 0.00 & 0.18 & 0.03 & 0.22 & 0.06 & 0.32 & 0.00 & 0.16 & 0.01 & 0.18 & 0.00 & 0.19 \\
\hline tilt & 0.15 & 1.73 & 0.40 & 3.95 & -0.03 & 1.17 & -0.65 & 3.13 & -0.04 & 1.44 & -0.76 & 3.01 & -0.02 & 1.14 & -0.06 & 1.19 & -0.06 & 1.88 \\
\hline slide & 0.19 & 0.49 & -0.24 & 0.43 & -0.02 & 0.27 & 0.06 & 0.27 & -0.30 & 0.26 & -0.28 & 0.43 & -0.09 & 0.28 & 0.01 & 0.28 & -0.36 & 0.28 \\
\hline rise & 3.22 & 0.10 & 3.20 & 0.39 & 3.27 & 0.07 & 3.27 & 0.08 & 3.30 & 0.10 & 3.31 & 0.14 & 3.26 & 0.07 & 3.26 & 0.08 & 3.28 & 0.08 \\
\hline roll & -0.96 & 4.24 & 2.14 & 4.49 & 2.84 & 2.14 & 3.03 & 2.67 & 1.52 & 2.18 & 1.78 & 3.07 & 1.74 & 2.22 & 1.73 & 2.27 & 0.90 & 2.72 \\
\hline twist & 37.27 & 4.28 & 31.46 & 5.62 & 34.73 & 1.33 & 34.63 & 1.82 & 34.75 & 1.39 & 32.19 & 2.82 & 35.03 & 1.33 & 35.06 & 1.37 & 34.79 & 2.87 \\
\hline xdisp & 0.36 & 0.58 & -1.02 & 0.84 & -0.59 & 0.51 & -0.43 & 0.52 & -0.85 & 0.54 & -1.12 & 0.87 & -0.53 & 0.53 & -0.38 & 0.53 & -0.83 & 0.52 \\
\hline ydisp & 0.10 & 0.27 & -0.12 & 0.70 & -0.01 & 0.27 & -0.01 & 0.30 & -0.05 & 0.50 & -0.09 & 0.59 & -0.01 & 0.27 & -0.01 & 0.28 & -0.01 & 0.29 \\
\hline helical-rise & 3.23 & 0.08 & 2.96 & 0.58 & 3.22 & 0.10 & 3.19 & 0.14 & 3.22 & 0.16 & 3.06 & 0.35 & 3.22 & 0.11 & 3.21 & 0.13 & 3.21 & 0.14 \\
\hline $\begin{array}{c}\text { helical- } \\
\text { inclination }\end{array}$ & -0.85 & 7.20 & 4.95 & 6.89 & 5.03 & 3.71 & 4.99 & 4.07 & 2.83 & 3.90 & 4.55 & 5.60 & 3.28 & 3.83 & 3.39 & 3.89 & 1.59 & 3.70 \\
\hline tip & -0.17 & 2.73 & 0.65 & 5.47 & 0.05 & 2.03 & 0.25 & 3.14 & 0.07 & 2.66 & 0.49 & 3.74 & 0.04 & 1.97 & 0.07 & 2.03 & 0.03 & 2.23 \\
\hline Helical-twist & 37.57 & 4.14 & 33.32 & 7.91 & 35.65 & 1.21 & 35.97 & 3.25 & 35.82 & 1.65 & 34.17 & 3.85 & 35.87 & 1.25 & 35.97 & 1.31 & 35.66 & 3.50 \\
\hline major width & 19.10 & 0.42 & 17.94 & 4.26 & 19.45 & 0.39 & 19.32 & 0.45 & 18.09 & 4.42 & 18.04 & 4.65 & 19.50 & 0.41 & 19.38 & 0.46 & 19.53 & 1.02 \\
\hline minor width & 12.20 & 0.33 & 11.21 & 2.73 & 12.17 & 0.26 & 12.10 & 0.30 & 11.30 & 2.89 & 11.24 & 2.97 & 12.19 & 0.26 & 12.13 & 0.30 & 12.22 & 0.64 \\
\hline pucker & 133.90 & 34.60 & 117.5 & 27.40 & 142.7 & 6.83 & 143.2 & 6.80 & 135.5 & 32.22 & 117.9 & 30.95 & 143.0 & 6.53 & 143.6 & 6.53 & 145.7 & 9.25 \\
\hline alpha & 274.20 & 66.90 & 284.7 & 11.32 & 291.0 & 3.98 & 290.1 & 4.98 & 286.4 & 5.87 & 287.9 & 7.03 & 291.7 & 4.08 & 290.6 & 5.28 & 286.8 & 6.35 \\
\hline beta & 176.40 & 19.90 & 169.0 & 4.05 & 171.8 & 3.72 & 168.8 & 5.04 & 166.3 & 6.03 & 169.1 & 3.84 & 172.7 & 3.88 & 170.4 & 5.23 & 167.2 & 5.89 \\
\hline gamma & 70.42 & 44.99 & 60.5 & 8.10 & 55.9 & 3.45 & 55.9 & 3.73 & 60.8 & 7.04 & 58.8 & 5.64 & 55.5 & 3.48 & 55.6 & 3.82 & 60.7 & 7.28 \\
\hline delta & 125.80 & 15.95 & 120.5 & 4.93 & 127.6 & 3.81 & 128.5 & 3.67 & 132.5 & 4.06 & 120.5 & 4.54 & 127.6 & 3.72 & 128.5 & 3.56 & 132.2 & 3.71 \\
\hline epsilon & 188.40 & 23.03 & 200.3 & 10.94 & 190.4 & 6.55 & 196.1 & 8.05 & 196.7 & 5.42 & 195.2 & 7.68 & 188.2 & 6.75 & 192.7 & 8.01 & 194.9 & 5.36 \\
\hline zeta & 257.40 & 32.17 & 248.6 & 16.87 & 254.3 & 6.94 & 248.1 & 8.32 & 251.3 & 6.57 & 255.9 & 10.08 & 255.5 & 7.05 & 250.5 & 7.82 & 252.2 & 6.46 \\
\hline chi & 247.80 & 16.23 & 241.4 & 7.13 & 249.6 & 3.94 & 250.1 & 4.88 & 245.9 & 4.10 & 242.2 & 6.35 & 249.2 & 3.94 & 250.3 & 3.86 & 245.4 & 4.03 \\
\hline
\end{tabular}


Table S5. Average structural parameters for the 3GGI systems.

\begin{tabular}{|c|c|c|c|c|c|c|c|c|c|c|c|c|c|c|c|c|c|c|}
\hline & & & \multicolumn{8}{|c|}{ Tip3p } & \multicolumn{8}{|c|}{ opc } \\
\hline & & & \multicolumn{2}{|c|}{ Bsc0 } & \multicolumn{2}{|c|}{$\varepsilon / \zeta_{\mathrm{OL} 1}+\mathrm{XOL4}_{4}$} & \multicolumn{2}{|c|}{ ol15 } & \multicolumn{2}{|c|}{ bsc1 } & \multicolumn{2}{|c|}{$\mathrm{Bsc0}$} & \multicolumn{2}{|c|}{$\varepsilon / \zeta_{\mathrm{OL} 1}+{ }^{\prime} \mathrm{OL} 4$} & \multicolumn{2}{|c|}{ ol15 } & \multicolumn{2}{|c|}{ bsc1 } \\
\hline & $\begin{array}{l}\text { Xray } \\
\text { Avg }\end{array}$ & $\begin{array}{c}\text { Xray Std } \\
\text { Dev }\end{array}$ & Avg & $\begin{array}{l}\text { Std } \\
\text { Dev }\end{array}$ & Avg & $\begin{array}{l}\text { Std } \\
\text { Dev }\end{array}$ & Avg & $\begin{array}{l}\text { Std } \\
\text { Dev }\end{array}$ & Avg & $\begin{array}{l}\text { Std } \\
\text { Dev }\end{array}$ & Avg & $\begin{array}{l}\text { Std } \\
\text { Dev }\end{array}$ & Avg & $\begin{array}{l}\text { Std } \\
\text { Dev }\end{array}$ & Avg & $\begin{array}{l}\text { Std } \\
\text { Dev }\end{array}$ & Avg & Std Dev \\
\hline shear & 0.00 & 0.21 & 0.01 & 0.21 & 0.00 & 0.13 & 0.00 & 0.12 & -0.01 & 0.19 & -0.01 & 0.17 & 0.02 & 0.18 & -0.01 & 0.11 & -0.02 & 0.20 \\
\hline stretch & -0.13 & 0.03 & -0.05 & 0.12 & -0.03 & 0.06 & -0.03 & 0.04 & -0.06 & 0.13 & -0.03 & 0.09 & -0.05 & 0.11 & -0.03 & 0.04 & -0.06 & 0.11 \\
\hline stagger & 0.15 & 0.14 & 0.06 & 0.22 & 0.09 & 0.18 & 0.12 & 0.17 & 0.14 & 0.18 & 0.11 & 0.22 & 0.09 & 0.17 & 0.12 & 0.16 & 0.14 & 0.17 \\
\hline buckle & 0.00 & 7.45 & -0.16 & 5.17 & -0.19 & 4.77 & -0.04 & 4.60 & 0.04 & 4.53 & 0.93 & 5.99 & -0.01 & 4.60 & 0.18 & 4.49 & 0.00 & 4.42 \\
\hline propeller & -8.72 & 2.54 & -5.82 & 4.79 & -8.10 & 3.42 & -9.04 & 3.07 & -6.44 & 3.45 & -6.10 & 5.06 & -8.19 & 3.19 & -8.65 & 2.96 & -6.58 & 3.18 \\
\hline opening & -1.55 & 1.64 & 0.71 & 2.72 & 0.08 & 1.73 & 0.04 & 1.52 & -0.11 & 1.76 & 0.50 & 4.05 & -0.04 & 1.60 & -0.05 & 1.54 & -0.16 & 1.56 \\
\hline shift & 0.00 & 0.34 & -0.10 & 0.43 & 0.01 & 0.25 & 0.00 & 0.20 & 0.00 & 0.24 & 0.08 & 0.36 & 0.00 & 0.19 & -0.01 & 0.19 & 0.02 & 0.24 \\
\hline tilt & 0.00 & 3.90 & 0.93 & 3.90 & 0.10 & 1.56 & 0.06 & 1.24 & 0.10 & 1.54 & -0.36 & 3.60 & 0.00 & 1.27 & -0.11 & 1.23 & -0.11 & 1.93 \\
\hline slide & 1.32 & 0.97 & -0.30 & 0.59 & -0.24 & 0.29 & -0.13 & 0.28 & -0.45 & 0.28 & -0.31 & 0.52 & -0.20 & 0.28 & -0.09 & 0.27 & -0.36 & 0.33 \\
\hline rise & 3.31 & 0.20 & 3.27 & 0.27 & 3.31 & 0.09 & 3.30 & 0.07 & 3.31 & 0.10 & 3.27 & 0.15 & 3.30 & 0.08 & 3.29 & 0.07 & 3.28 & 0.12 \\
\hline roll & 1.39 & 6.34 & 1.96 & 4.13 & 2.62 & 2.81 & 2.63 & 2.21 & 2.27 & 2.45 & 3.67 & 3.50 & 3.07 & 2.08 & 2.60 & 2.10 & 2.51 & 2.69 \\
\hline twist & 37.29 & 12.02 & 29.34 & 4.86 & 33.68 & 1.62 & 33.86 & 1.55 & 33.44 & 1.90 & 29.10 & 4.14 & 34.32 & 1.65 & 34.19 & 1.53 & 34.10 & 3.00 \\
\hline xdisp & 1.00 & 2.07 & -2.01 & 1.43 & -1.05 & 0.69 & -0.79 & 0.69 & -1.27 & 0.68 & -2.26 & 1.53 & -0.97 & 0.64 & -0.71 & 0.67 & -1.20 & 0.67 \\
\hline ydisp & 0.00 & 1.55 & 0.14 & 0.94 & 0.00 & 0.37 & 0.01 & 0.34 & 0.01 & 0.40 & -0.04 & 0.94 & 0.00 & 0.33 & -0.01 & 0.35 & -0.01 & 0.37 \\
\hline helical-rise & 3.15 & 0.28 & 2.90 & 0.47 & 3.16 & 0.22 & 3.20 & 0.12 & 3.21 & 0.16 & 2.96 & 0.33 & 3.20 & 0.12 & 3.19 & 0.12 & 3.18 & 0.13 \\
\hline $\begin{array}{c}\text { helical- } \\
\text { inclination }\end{array}$ & 5.56 & 11.85 & 3.06 & 7.14 & 5.01 & 4.73 & 5.04 & 4.09 & 4.26 & 4.19 & 4.61 & 7.06 & 5.54 & 3.71 & 4.92 & 3.83 & 4.72 & 3.92 \\
\hline tip & 0.00 & 6.88 & -1.14 & 5.05 & -0.15 & 2.57 & -0.10 & 2.27 & -0.12 & 2.54 & 0.35 & 4.89 & -0.01 & 2.24 & 0.18 & 2.23 & 0.01 & 2.43 \\
\hline Helical-twist & 38.19 & 11.50 & 31.70 & 6.15 & 34.98 & 1.63 & 34.88 & 1.42 & 34.53 & 2.11 & 30.78 & 5.91 & 35.28 & 1.55 & 35.14 & 1.41 & 35.17 & 3.43 \\
\hline major width & 18.15 & 0.71 & 18.05 & 1.81 & 19.34 & 0.70 & 19.36 & 0.30 & 19.44 & 0.83 & 18.62 & 1.31 & 19.38 & 0.46 & 19.29 & 0.33 & 19.38 & 0.91 \\
\hline minor width & 11.71 & 0.33 & 11.39 & 1.09 & 12.15 & 0.42 & 12.19 & 0.16 & 12.21 & 0.51 & 11.75 & 0.78 & 12.18 & 0.27 & 12.16 & 0.18 & 12.17 & 0.57 \\
\hline pucker & 151.8 & 17.73 & 125.1 & 15.99 & 145.3 & 7.78 & 147.8 & 6.33 & 147.5 & 8.63 & 131.5 & 15.59 & 147.4 & 6.87 & 148.9 & 6.25 & 148.6 & 8.73 \\
\hline alpha & 289.9 & 5.34 & 277.6 & 11.61 & 288.4 & 5.12 & 287.9 & 5.05 & 283.5 & 6.76 & 278.7 & 10.93 & 289.1 & 4.67 & 288.1 & 5.31 & 283.5 & 6.97 \\
\hline beta & 157.9 & 18.52 & 170.3 & 4.19 & 171.0 & 3.88 & 167.2 & 5.28 & 166.9 & 5.75 & 170.5 & 4.08 & 171.2 & 3.88 & 167.8 & 5.18 & 167.2 & 5.52 \\
\hline gamma & 47.7 & 5.18 & 63.1 & 9.53 & 55.3 & 4.53 & 54.1 & 4.10 & 60.2 & 7.99 & 61.6 & 9.04 & 54.9 & 4.13 & 54.2 & 4.07 & 59.6 & 7.73 \\
\hline delta & 135.1 & 9.48 & 122.5 & 5.01 & 130.6 & 4.02 & 132.2 & 3.71 & 134.5 & 3.93 & 123.1 & 4.91 & 131.5 & 3.86 & 132.8 & 3.61 & 135.3 & 3.70 \\
\hline epsilon & 224.5 & 40.85 & 206.4 & 10.68 & 195.3 & 7.09 & 202.1 & 8.22 & 200.1 & 5.53 & 201.5 & 9.65 & 193.9 & 6.73 & 200.1 & 7.74 & 199.8 & 5.40 \\
\hline zeta & 218.5 & 56.86 & 239.3 & 17.01 & 247.7 & 9.56 & 240.8 & 8.85 & 247.7 & 7.93 & 249.1 & 14.11 & 248.8 & 8.34 & 241.8 & 8.38 & 246.8 & 7.46 \\
\hline chi & 266.5 & 12.79 & 240.1 & 10.27 & 249.3 & 4.51 & 251.3 & 4.05 & 245.8 & 4.42 & 241.9 & 9.18 & 250.7 & 4.08 & 252.4 & 3.96 & 246.7 & 4.47 \\
\hline
\end{tabular}


Table S6. CPPTRAJ input commands for selected analysis. All cpptraj analysis was done using version 15.0 available in freely in AmberTools14 (ambermd.org) or from the GitHub website (https://github.com/Amber-MD/cpptraj).

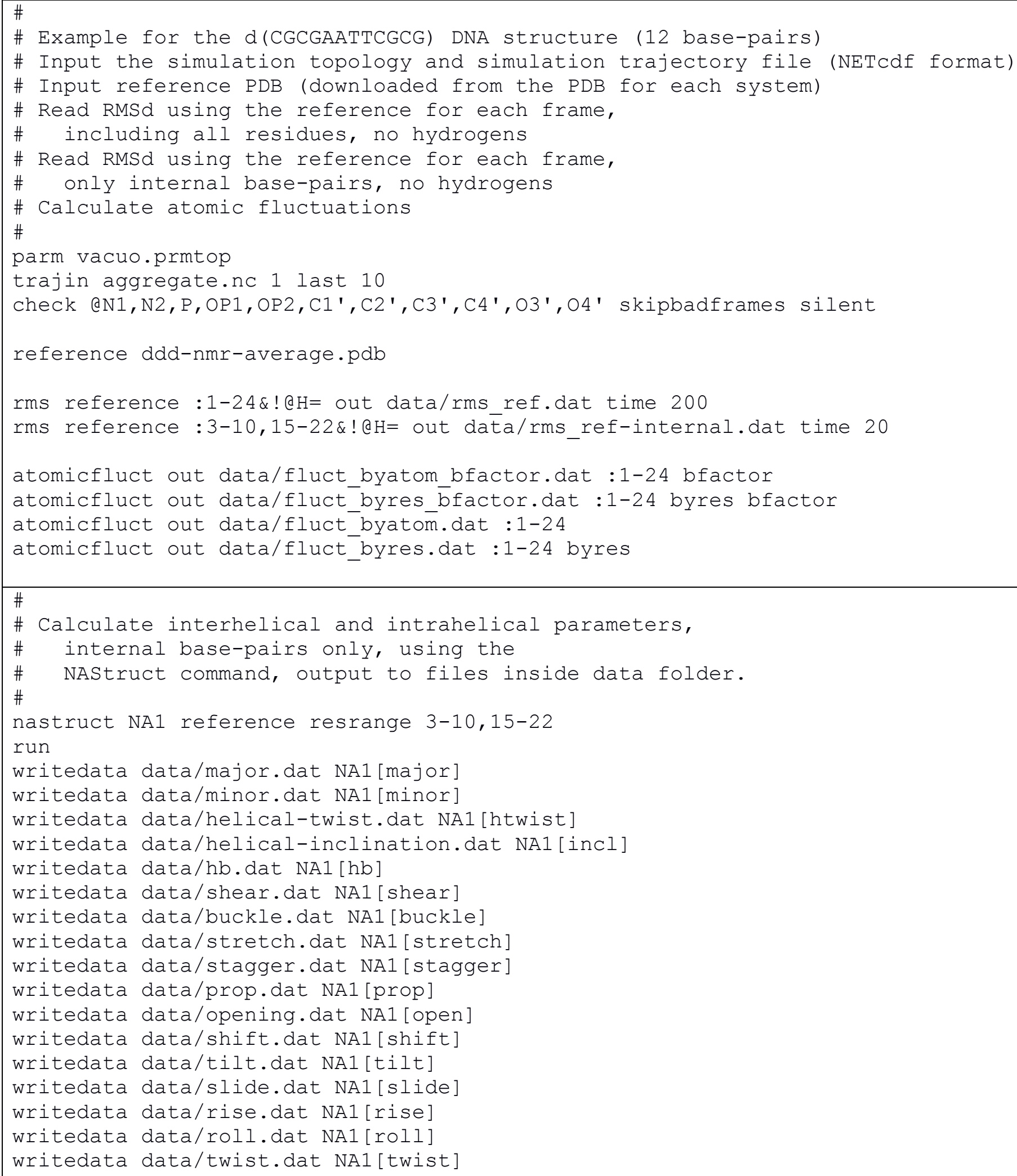


Table S7. Root mean square deviations (RMSD) of average structures from molecular dynamics simulations to reference structure of each system $(\AA)$. The average structures from simulations were calculated over the full aggregated trajectories of each system. The DDD and 1FZX references were NMR averages; the reference structures for 1SK5, 3GGI, and 1I0T were X-ray structures. RMSDs were calculated over all heavy atoms of the internal eight base pairs. The $\varepsilon / \xi_{\mathrm{OL} 1 \mathrm{XOL}}$ includes bsc0 corrections.

\begin{tabular}{|l|c|c|c|c|c|c|c|c|}
\hline & \multicolumn{4}{|c|}{ TIP3P } & \multicolumn{4}{c|}{ OPC } \\
\hline & bsc0 & $\boldsymbol{\varepsilon} /$ SOL1XoL4 $_{\text {OLI }}$ & OL15 & bsc1 & bsc0 & $\boldsymbol{\varepsilon} / \boldsymbol{\xi}_{\text {OL1XoL4 }}$ & OL15 & bsc1 \\
\hline DDD & 1.00 & 0.55 & 0.53 & 0.64 & 0.91 & 0.44 & 0.44 & 0.61 \\
\hline 1FZX & 1.04 & 0.78 & 0.83 & 0.87 & 0.90 & 0.73 & 0.77 & 0.80 \\
\hline 1SK5 & 1.61 & 1.15 & 1.14 & 1.34 & 1.66 & 1.05 & 1.04 & 1.21 \\
\hline 3GGI & 2.40 & 2.24 & 2.02 & 2.30 & 2.62 & 2.22 & 1.99 & 2.28 \\
\hline 110T & 0.93 & 0.95 & 0.94 & 1.19 & 0.95 & 0.93 & 0.94 & 1.15 \\
\hline
\end{tabular}


Table S8. Average structural parameters for the DDD simulations using the CHARMM 36 force field. The CHARMM-JC refers to the Joung-Cheatham ion model. The two terminal base pairs on each side of the DDD were excluded for the average value calculation.

\begin{tabular}{|r|c|c|c|c|c|c|}
\hline & & & CHARMM & & \multicolumn{2}{c|}{ CHARMM-JC } \\
\hline & NMR Avg. & NMR Std. Dev & Avg. & Std. Dev. & Avg. & Std. Dev. \\
\hline shear & 0 & 0.24 & 0 & 0.13 & 0 & 0.13 \\
\hline stretch & -0.34 & 0.09 & -0.1 & 0.09 & -0.1 & 0.11 \\
\hline stagger & -0.12 & 0.11 & -0.14 & 0.2 & -0.14 & 0.21 \\
\hline buckle & -0.02 & 2.91 & -0.01 & 5.25 & 0.08 & 5.3 \\
\hline propeller & -17.58 & 3.93 & -11.49 & 4.07 & -11.31 & 4.13 \\
\hline opening & -1.10 & 1.21 & 0.68 & 2.46 & 0.68 & 2.56 \\
\hline shift & 0.00 & 0.32 & 0 & 0.17 & 0.01 & 0.17 \\
\hline tilt & 0.01 & 1.09 & -0.01 & 1.36 & 0.04 & 1.37 \\
\hline slide & -0.21 & 0.18 & 0.33 & 0.32 & 0.34 & 0.32 \\
\hline rise & 3.20 & 0.16 & 3.3 & 0.3 & 3.27 & 0.39 \\
\hline roll & 3.03 & 3.54 & 5.91 & 2.36 & 5.76 & 2.49 \\
\hline twist & 35.71 & 1.91 & 32.87 & 3.44 & 32.55 & 4.25 \\
\hline x-disp & -0.81 & 0.35 & -0.45 & 0.76 & -0.4 & 0.76 \\
\hline y-disp & 0.00 & 0.45 & 0 & 0.36 & 0 & 0.37 \\
\hline helical-rise & 3.18 & 0.12 & 3.2 & 0.3 & 3.18 & 0.39 \\
\hline helical-inclination & 5.04 & 5.93 & 10.42 & 4.26 & 10.2 & 4.41 \\
\hline tip & -0.01 & 1.87 & 0.01 & 2.52 & -0.07 & 2.54 \\
\hline helical-twist & 36.03 & 1.71 & 34.57 & 3.58 & 34.24 & 4.45 \\
\hline Major groove & 19.56 & 0.22 & 18.42 & 1.29 & 18.28 & 1.86 \\
\hline Minor groove & 12.22 & 0.25 & 12.01 & 0.84 & 11.92 & 1.22 \\
\hline pucker & 137.10 & 14.22 & 134.44 & 12.22 & 134.44 & 12.35 \\
\hline alpha & 298.80 & 6.19 & 295.55 & 10.35 & 294.96 & 11.89 \\
\hline beta & 172.40 & 4.90 & 158.68 & 6.74 & 158.52 & 7.13 \\
\hline gamma & 50.28 & 4.92 & 51.88 & 4.37 & 51.9 & 4.45 \\
\hline chilan & 126.70 & 9.00 & 126.87 & 5.28 & 126.91 & 5.36 \\
\hline & 188.50 & 5.16 & 207.62 & 10.15 & 208.29 & 9.94 \\
\hline & 257.10 & 11.96 & 238.21 & 13.12 & 237.16 & 13.47 \\
\hline & 7.85 & 250.61 & 6.85 & 250.89 & 7.13 \\
\hline
\end{tabular}


Table S9. Average $p$-values for DNA helical parameters of DDD. The $p$-values were calculated from a twosample t-test; values above 0.05 indicate that the null hypothesis is accepted and the differences between the sample means are not significant. P-values below 0.05 indicate that the null hypothesis is rejected and that sample means are different. The t-tests were performed for each base, base pair, or base step (depending on the property) and the average $p$-value comes from averaging across the internal eight base-pairs of DDD. The t-tests were calculated to compare the force fields (e.g. bsc1-OL15) and for experimental data from NMR and the force fields (e.g. NMR-OL15).

\begin{tabular}{|c|c|c|c|c|c|c|}
\hline & \multicolumn{3}{|c|}{ TIP3P } & \multicolumn{3}{|c|}{ OPC } \\
\hline & bsc1-OL15 & NMR-bsc1 & NMR-OL15 & bsc1-OL15 & NMR-bsc1 & NMR-OL15 \\
\hline Alpha & $2.20 E-16$ & 0.00 & 0.05 & $2.20 \mathrm{E}-16$ & 0.00 & 0.11 \\
\hline Beta & $2.20 \mathrm{E}-16$ & 0.00 & 0.03 & $2.20 \mathrm{E}-16$ & 0.00 & 0.11 \\
\hline Gamma & $2.20 \mathrm{E}-16$ & 0.01 & 0.11 & $2.20 \mathrm{E}-16$ & 0.08 & 0.12 \\
\hline Delta & $2.20 \mathrm{E}-16$ & 0.00 & 0.12 & $2.20 \mathrm{E}-16$ & 0.00 & 0.11 \\
\hline Epsilon & $2.20 \mathrm{E}-16$ & 2.52E-05 & 2.39E-05 & $2.20 \mathrm{E}-16$ & 2.73E-04 & 3.21E-04 \\
\hline Zeta & $2.20 \mathrm{E}-16$ & 1.36E-05 & 8.95E-05 & $2.20 \mathrm{E}-16$ & 5.62E-06 & 2.20E-02 \\
\hline Chi & $2.20 \mathrm{E}-16$ & 0.02 & 0.04 & $2.20 \mathrm{E}-16$ & 0.00 & 0.11 \\
\hline Shear & 3.81E-03 & 0.05 & 0.05 & 8.64E-04 & 0.04 & 0.05 \\
\hline Stagger & $2.20 \mathrm{E}-16$ & 0.16 & 0.02 & $2.20 \mathrm{E}-16$ & 0.17 & 0.03 \\
\hline Propeller & $2.20 \mathrm{E}-16$ & 8.60E-05 & 0.00 & 8.64E-04 & 0.00 & 0.00 \\
\hline Stretch & $2.20 \mathrm{E}-16$ & 4.25E-04 & 3.98E-04 & $2.20 \mathrm{E}-16$ & 0.00 & 4.21E-04 \\
\hline Buckle & $3.72 \mathrm{E}-02$ & $6.54 \mathrm{E}-04$ & $6.00 \mathrm{E}-04$ & $1.42 \mathrm{E}-03$ & 1.08E-03 & 1.61E-03 \\
\hline Opening & 5.21E-04 & 0.12 & 0.02 & $2.20 \mathrm{E}-16$ & 1.02E-01 & 0.03 \\
\hline Shift & 0.05 & 0.09 & 0.11 & 0.00 & 0.34 & 0.35 \\
\hline Slide & $2.20 \mathrm{E}-16$ & 0.02 & 0.16 & $2.20 \mathrm{E}-16$ & 0.24 & 0.00 \\
\hline Roll & $2.20 \mathrm{E}-16$ & 0.02 & 0.03 & 1.29E-05 & 0.04 & 0.09 \\
\hline Tilt & 5.51E-02 & 0.12 & 0.27 & 1.31E-01 & 0.28 & 0.39 \\
\hline Rise & $4.26 \mathrm{E}-12$ & $6.20 \mathrm{E}-05$ & 0.00 & $2.20 \mathrm{E}-16$ & 6.59E-05 & 0.00 \\
\hline Twist & $2.20 \mathrm{E}-16$ & 0.01 & 0.01 & $1.05 E-04$ & 0.01 & 0.01 \\
\hline
\end{tabular}

Table S10. Ability of each force field to reproduce NOE based distance restraints (125 restraints, based on the 1 NAJ structure). The number corresponds of the average number of violations for each restraint divided by the total number of analyzed frames (std. dev. In parenthesis).

\begin{tabular}{|l|c|c|c|c|c|c|}
\hline & \multicolumn{3}{|c|}{ OPC } & \multicolumn{3}{c|}{ TIP3P } \\
\hline & Bsc0 & OL15 & Bsc1 & Bsc0 & OL15 & Bsc1 \\
\hline Fraction of violated restraints & $0.40( \pm 0.2)$ & $0.29( \pm 0.1)$ & $0.37( \pm 0.2)$ & $0.37( \pm 0.1)$ & $0.33( \pm 0.2)$ & $0.34( \pm 0.1)$ \\
\hline
\end{tabular}


Figure S1. DDD average structures calculated from the aggregated trajectories aligned to the NMR average structure using only the heavy atoms. The detail represents A6 (shown in a slightly thicker representation in the full helix). The RMSD values of the heavy atoms of the internal eight base pairs from the MD average structures to the NMR average structure are less than $1 \AA$ for all three force fields in both water models.
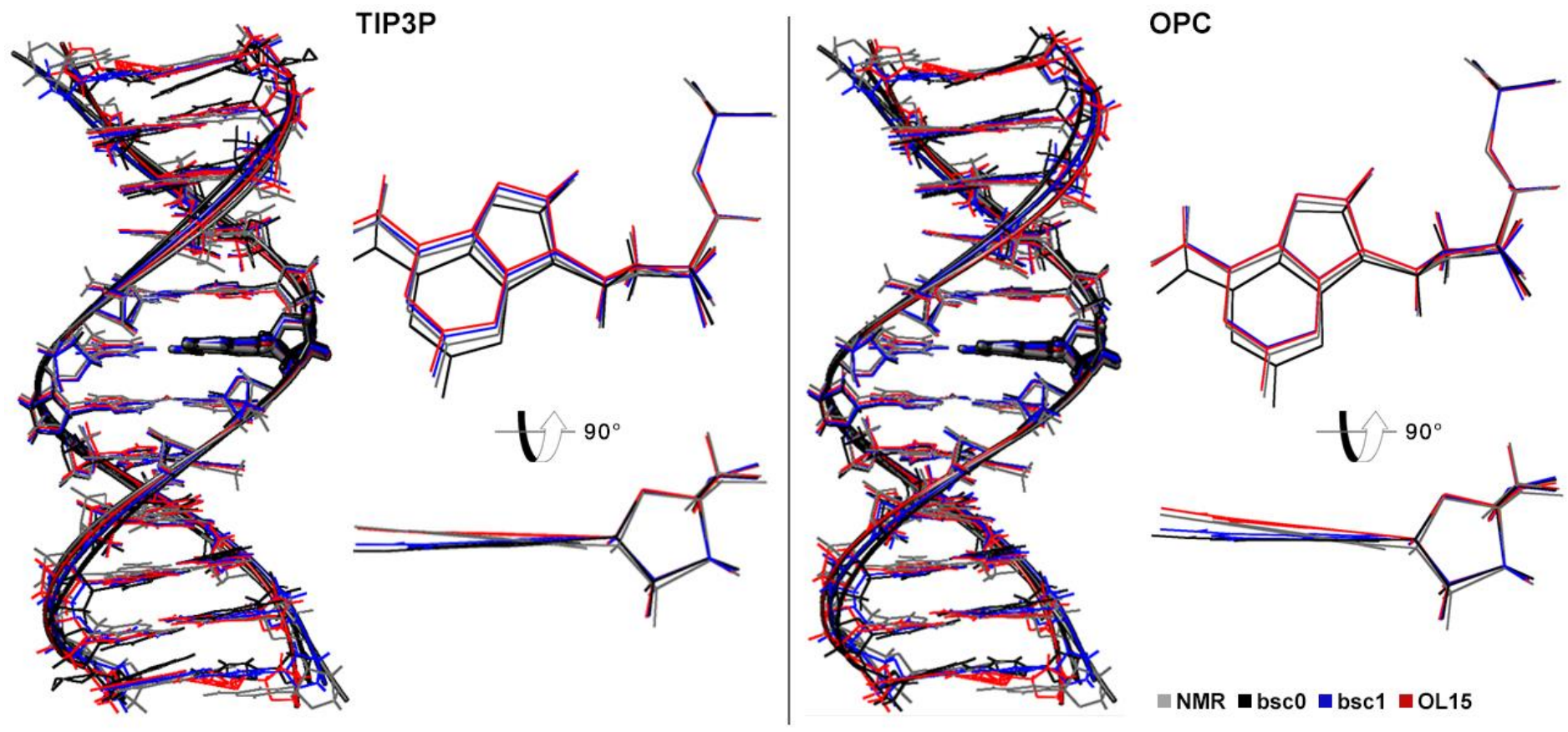

NMR $\square$ bsc0 $\square$ bsc1 $\approx$ OL15 
Figure S2. DDD overlay of NMR average structure (dark grey) with the average structure of the full trajectory for CHARMM36 and CHARMM36 with the Joung-Cheatham ions. Alignment done using the heavy atoms of internal residues. The detail represents A6 (shown in a slightly thicker representation in the full helix).

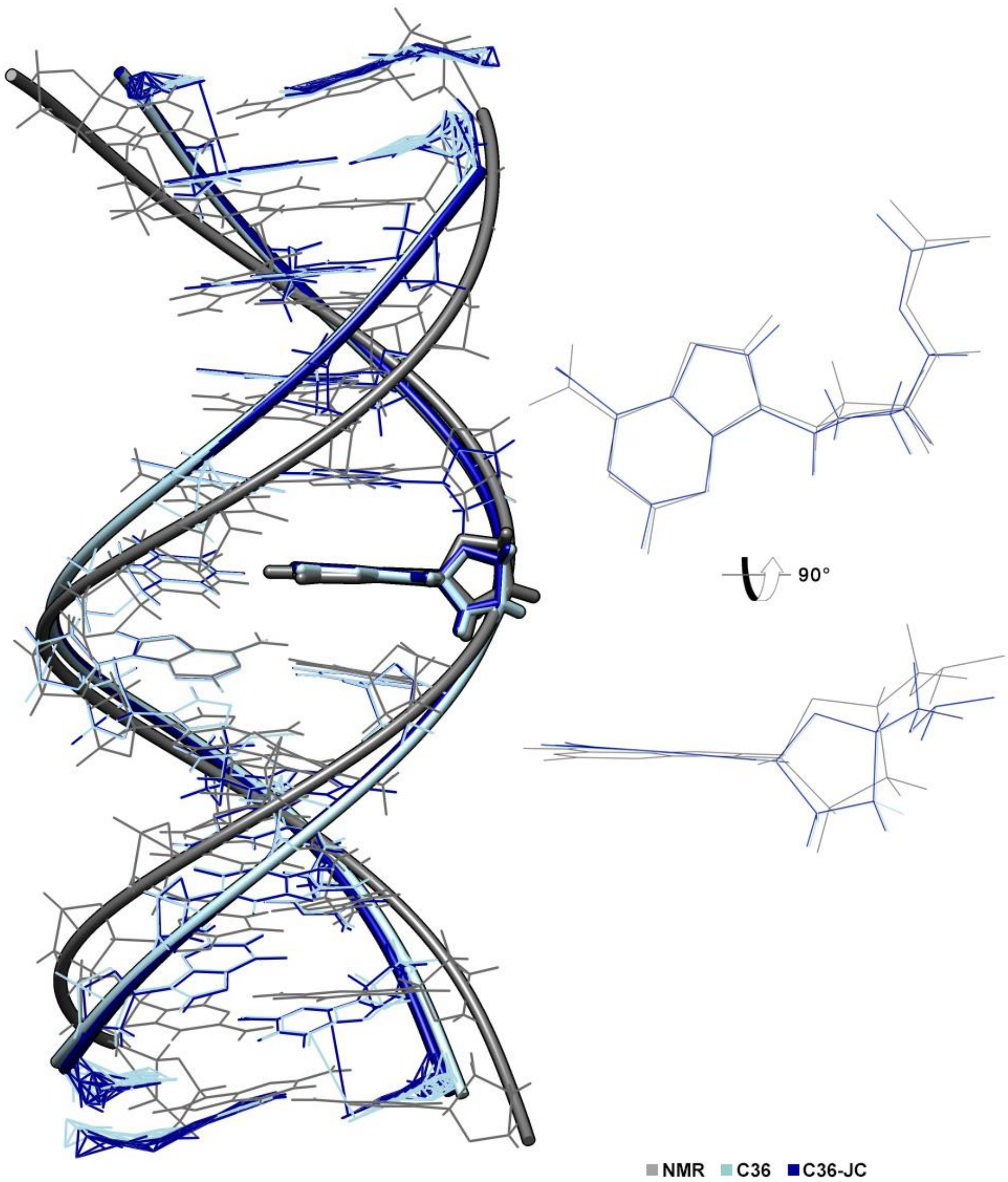


Figure S3. Selected global helical parameters for individual base pairs steps in the DDD dodecamer.

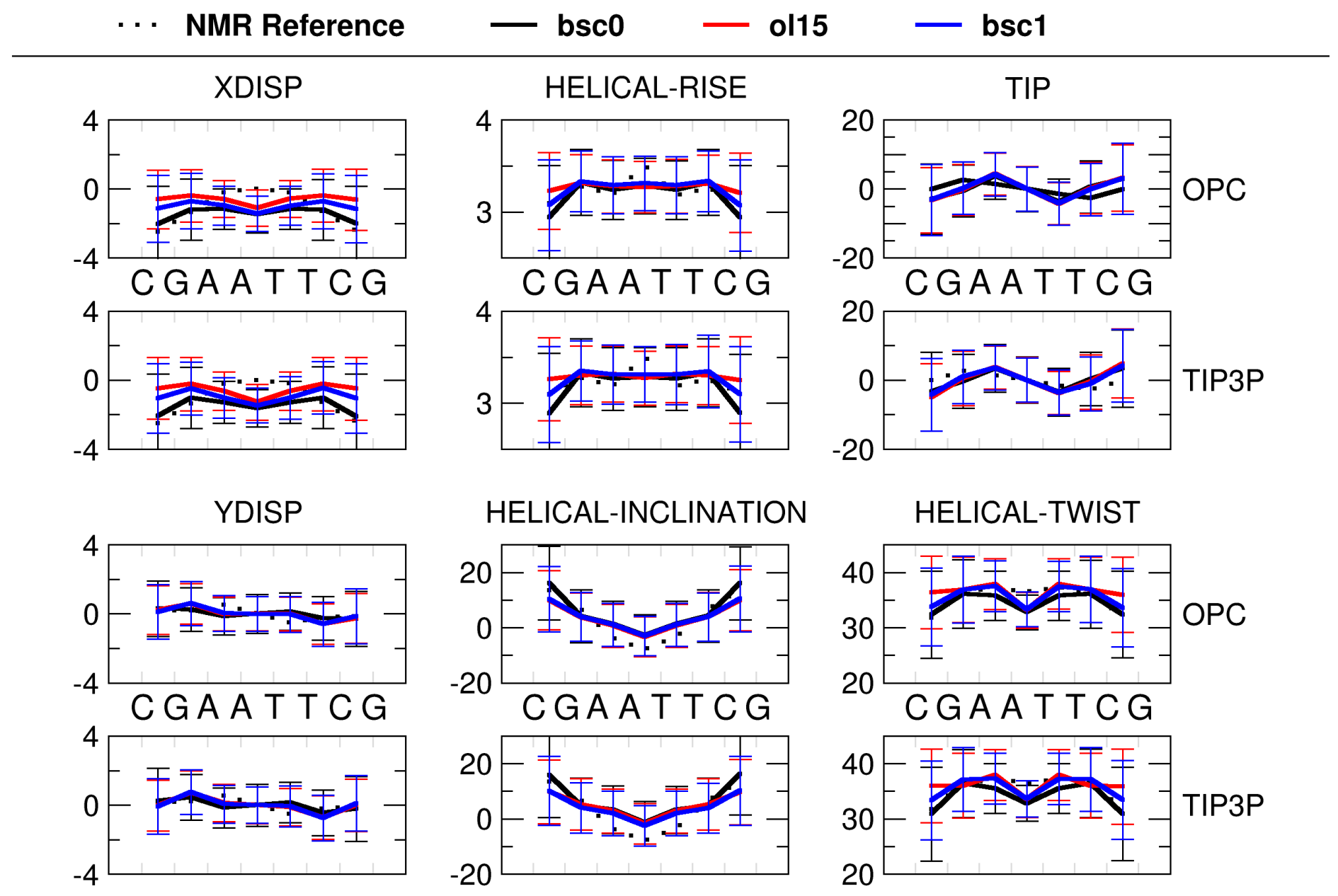


Figure S4. Selected intra-base helical parameters for individual base pairs steps in the DDD dodecamer.

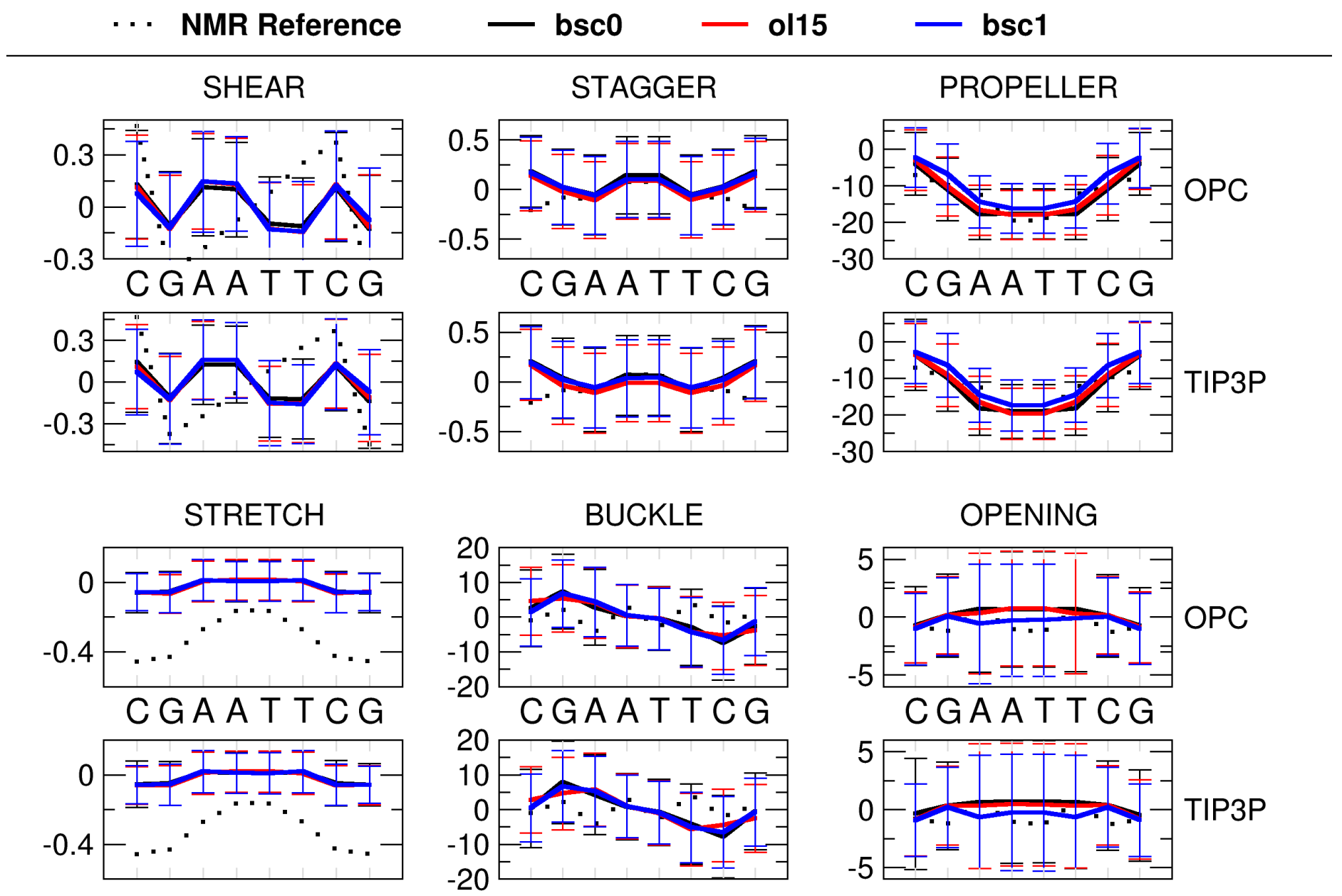


Figure S5. Selected inter-base helical parameters for individual base pairs steps in the DDD dodecamer.

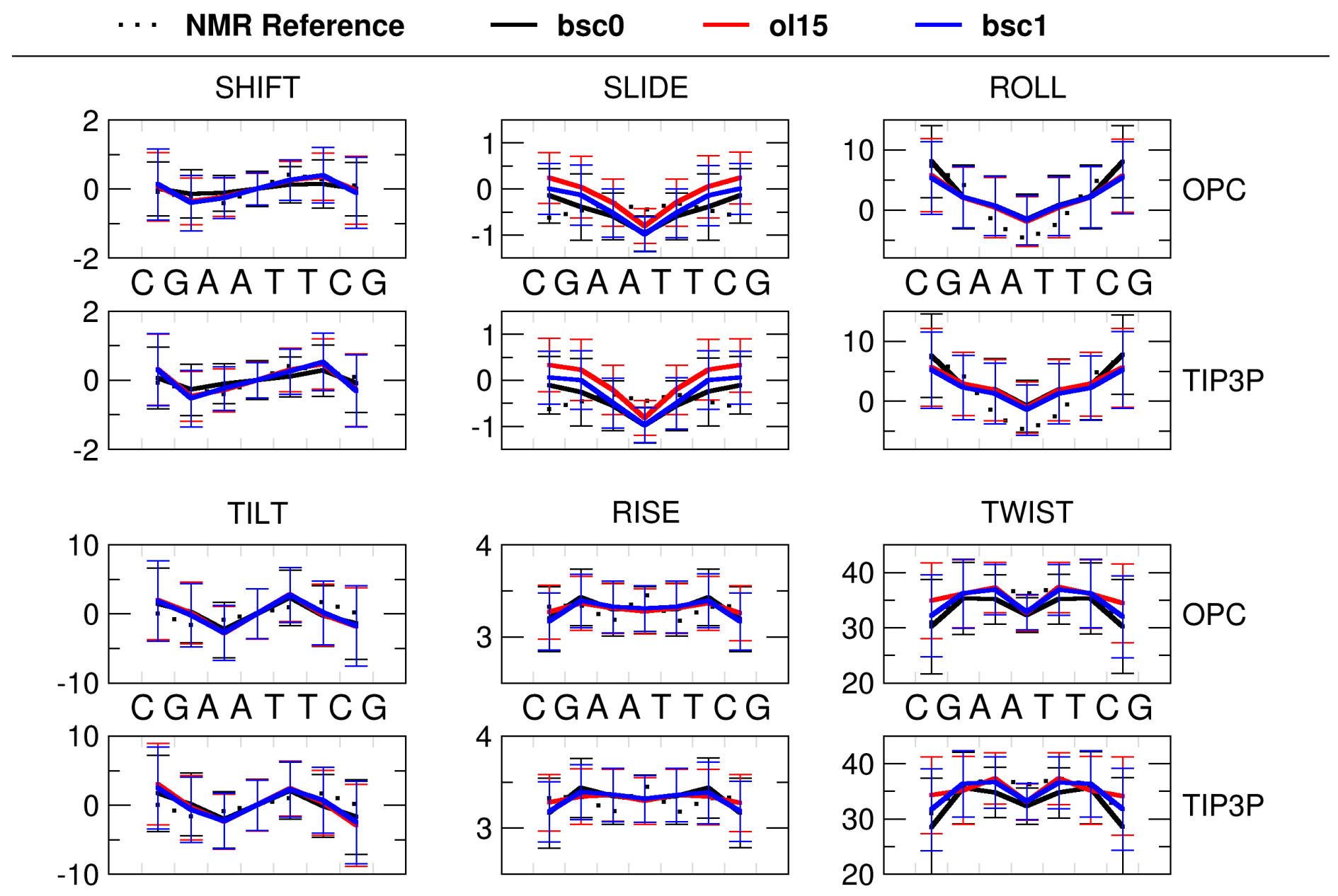


Figure S6. Selected groove widths for the DDD dodecamer.

.. NMR Reference - bsc0 - ol15 - bsc1

\section{MAJOR GROOVE WIDTH}

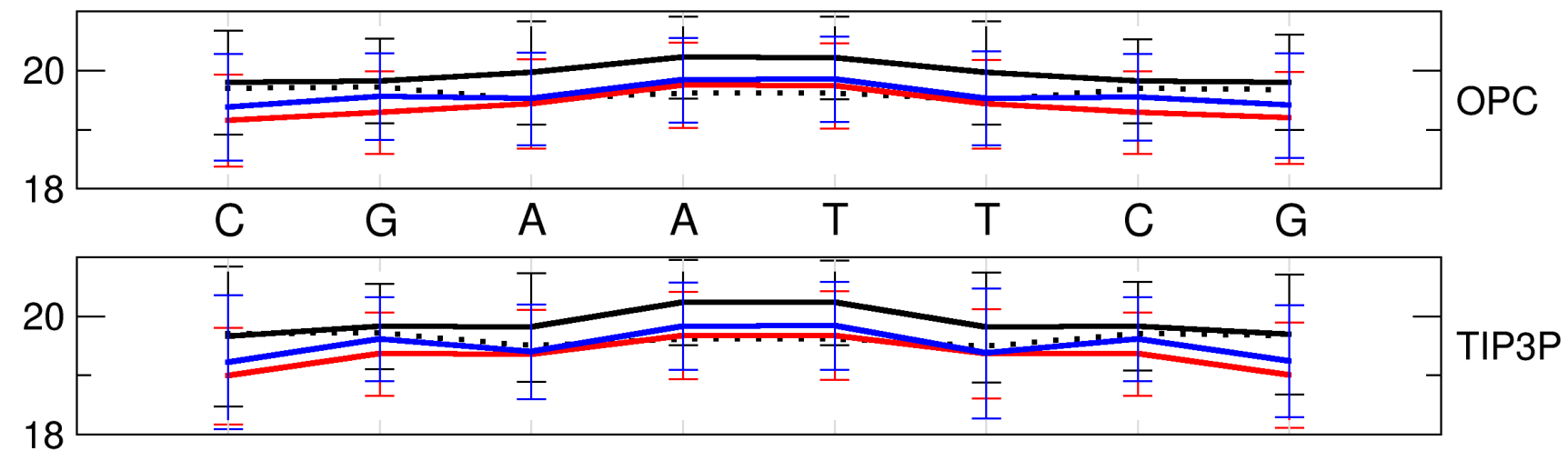

MINOR GROOVE WIDTH

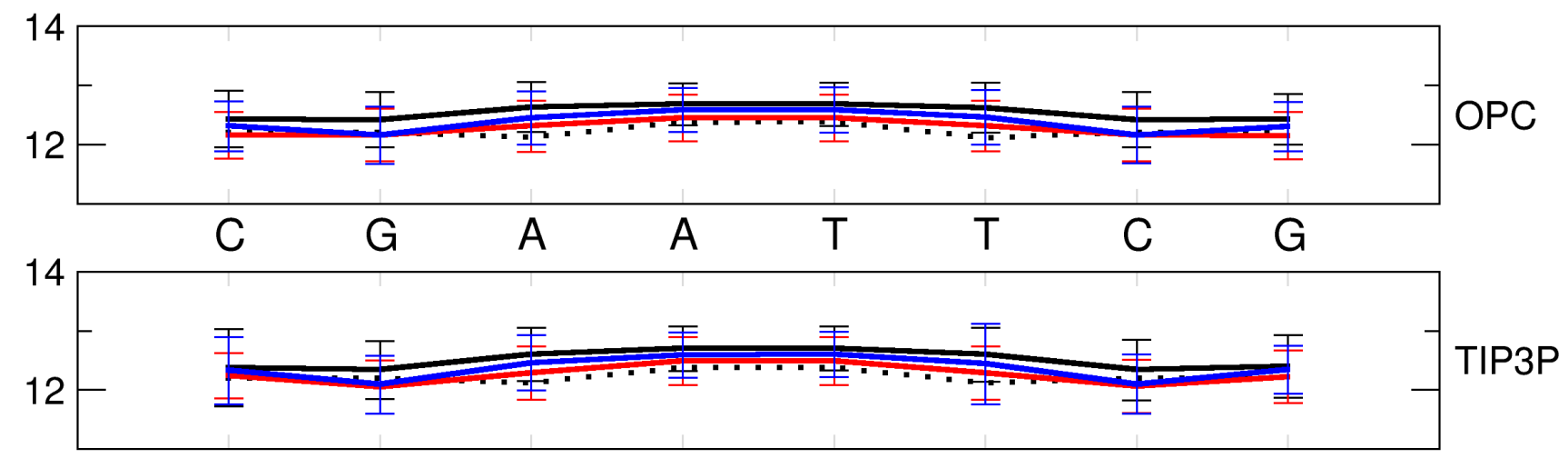


Figure S7. Selected global helical parameters for individual base pairs steps in the 1FZX system.

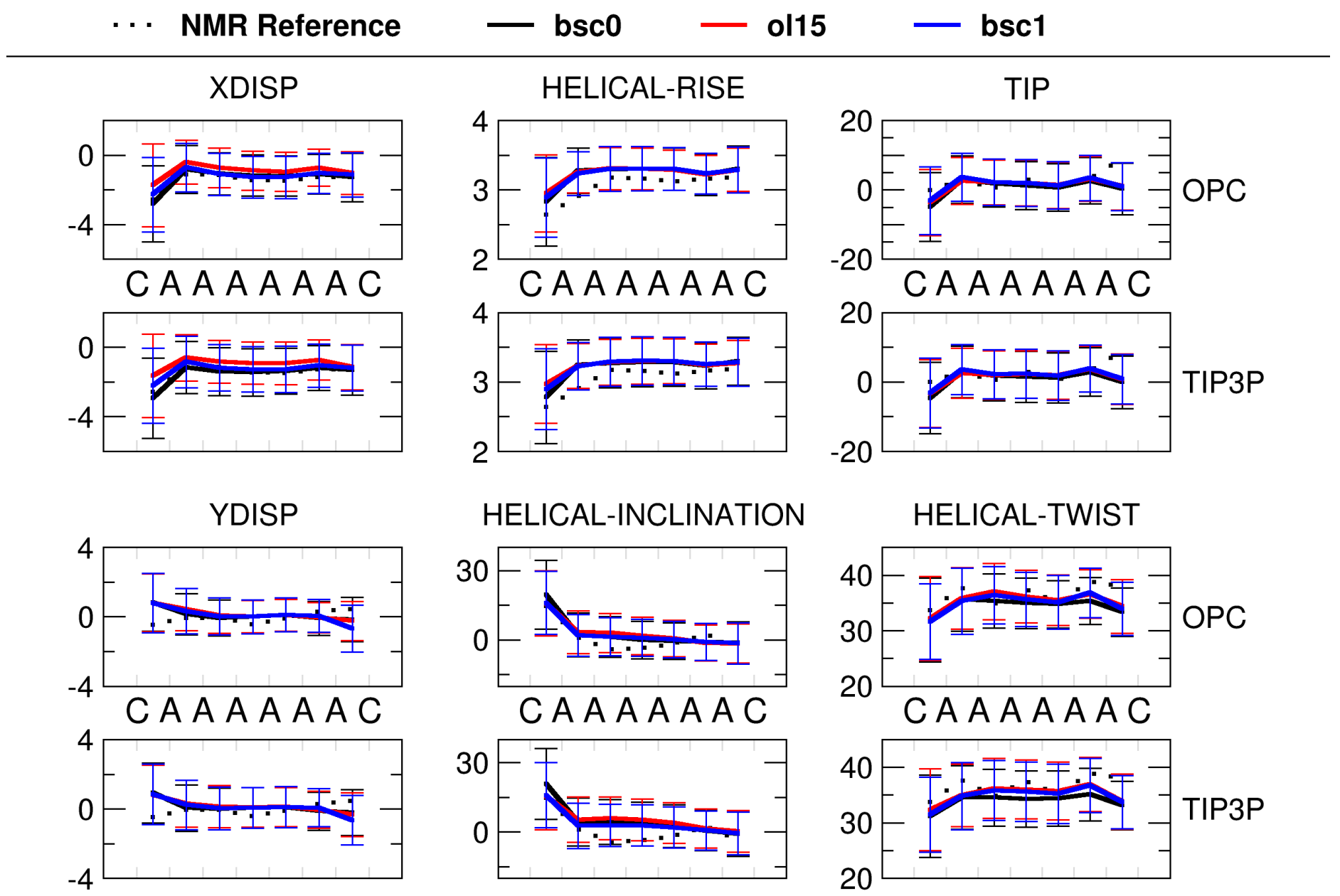


Figure S8. Selected intra-base helical parameters for individual base pairs steps in the 1FZX system

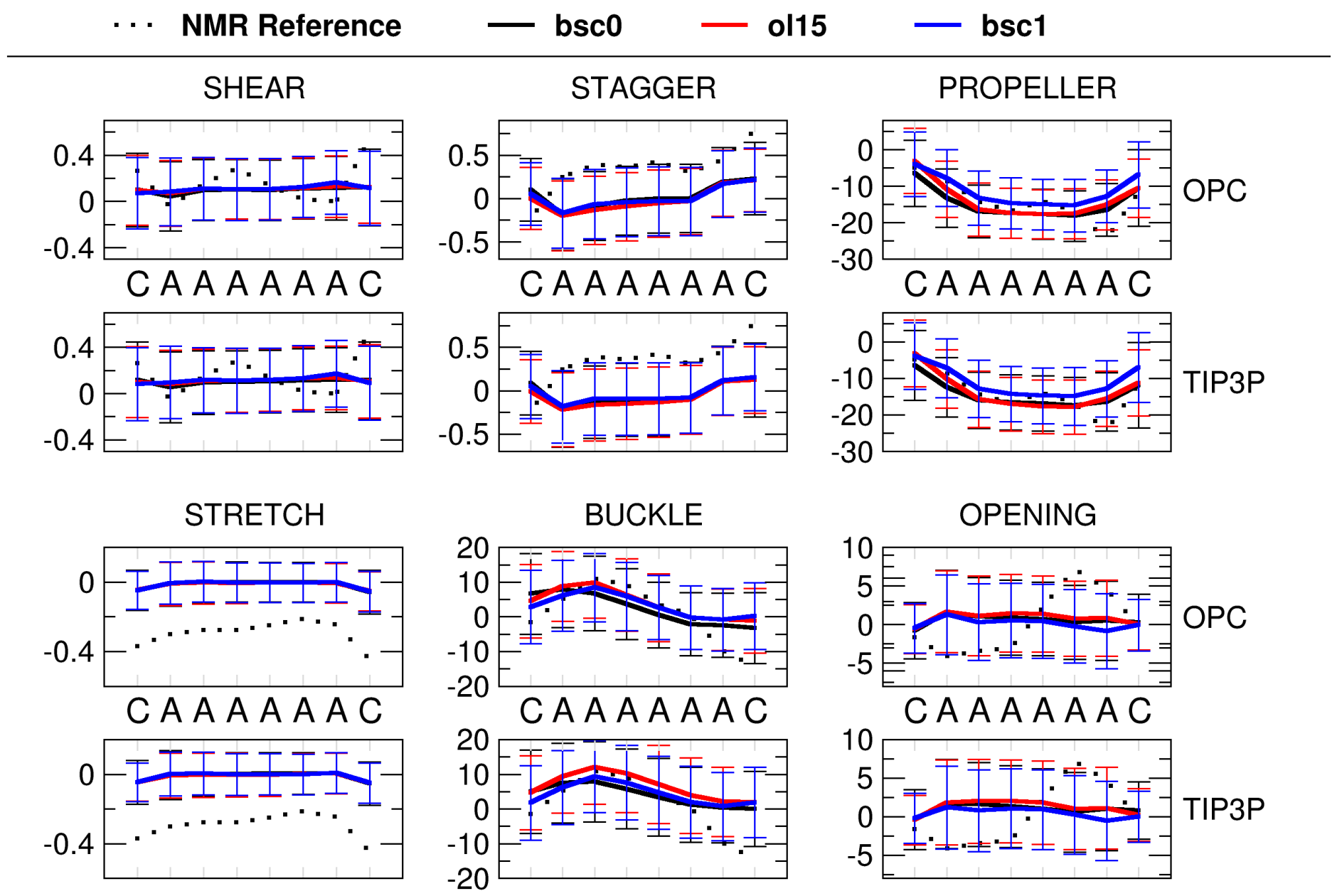


Figure S9. Selected inter-base helical parameters for individual base pairs steps in the 1FZX system.

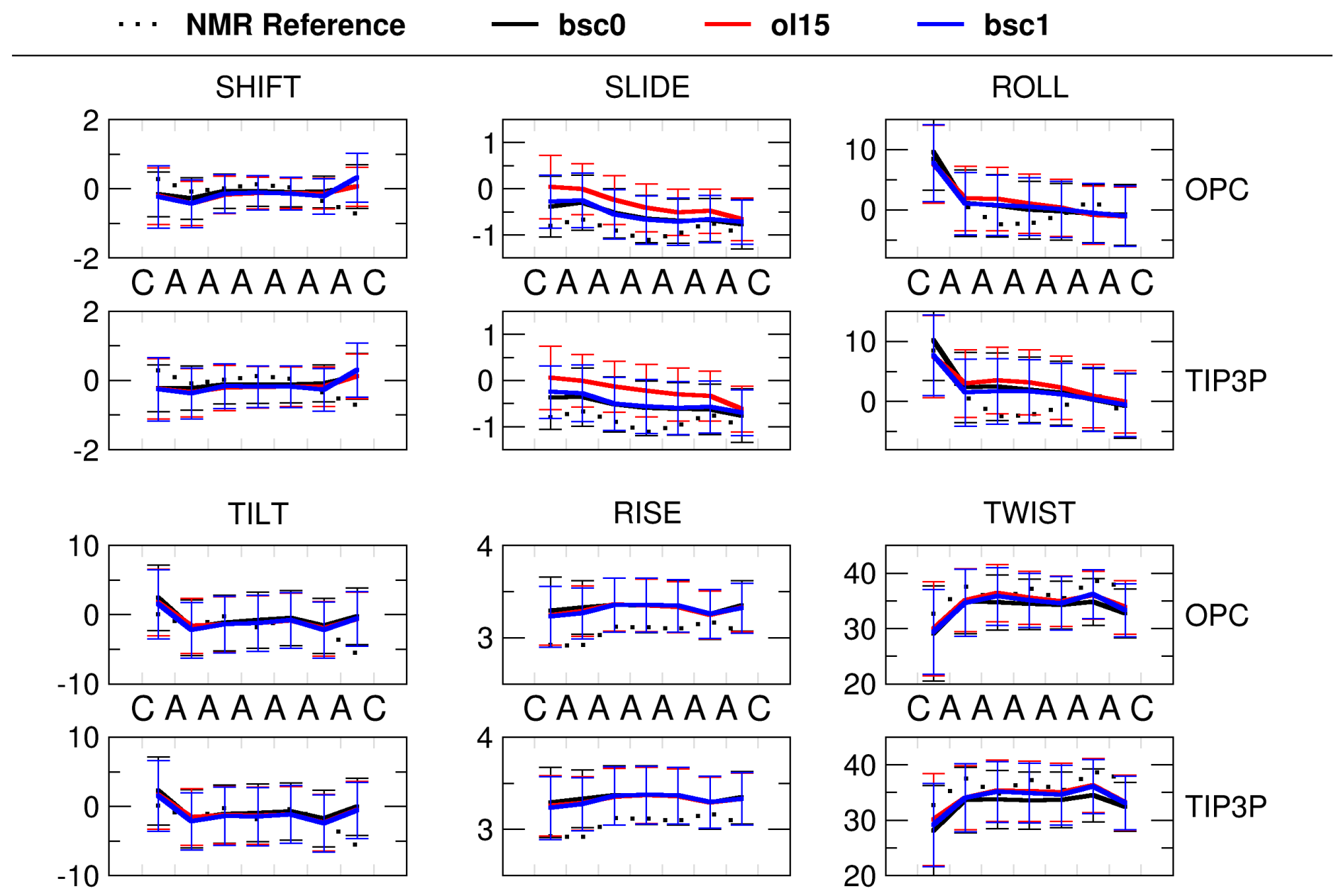


Figure S10. Selected groove widths for the $1 \mathrm{FXZ}$.

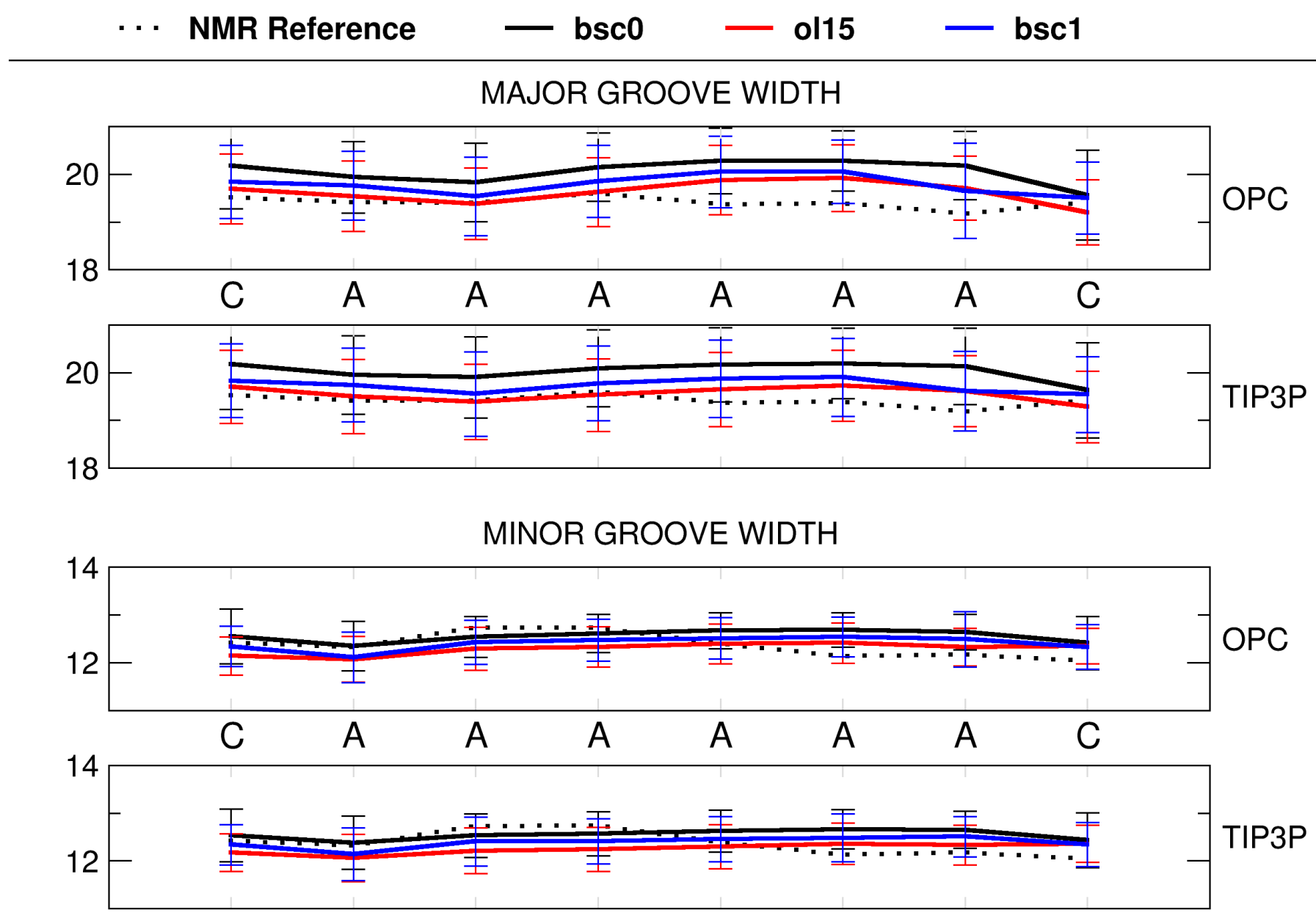


Figure S11. Selected global helical parameters for individual base pairs steps in the 1 SK5 system.

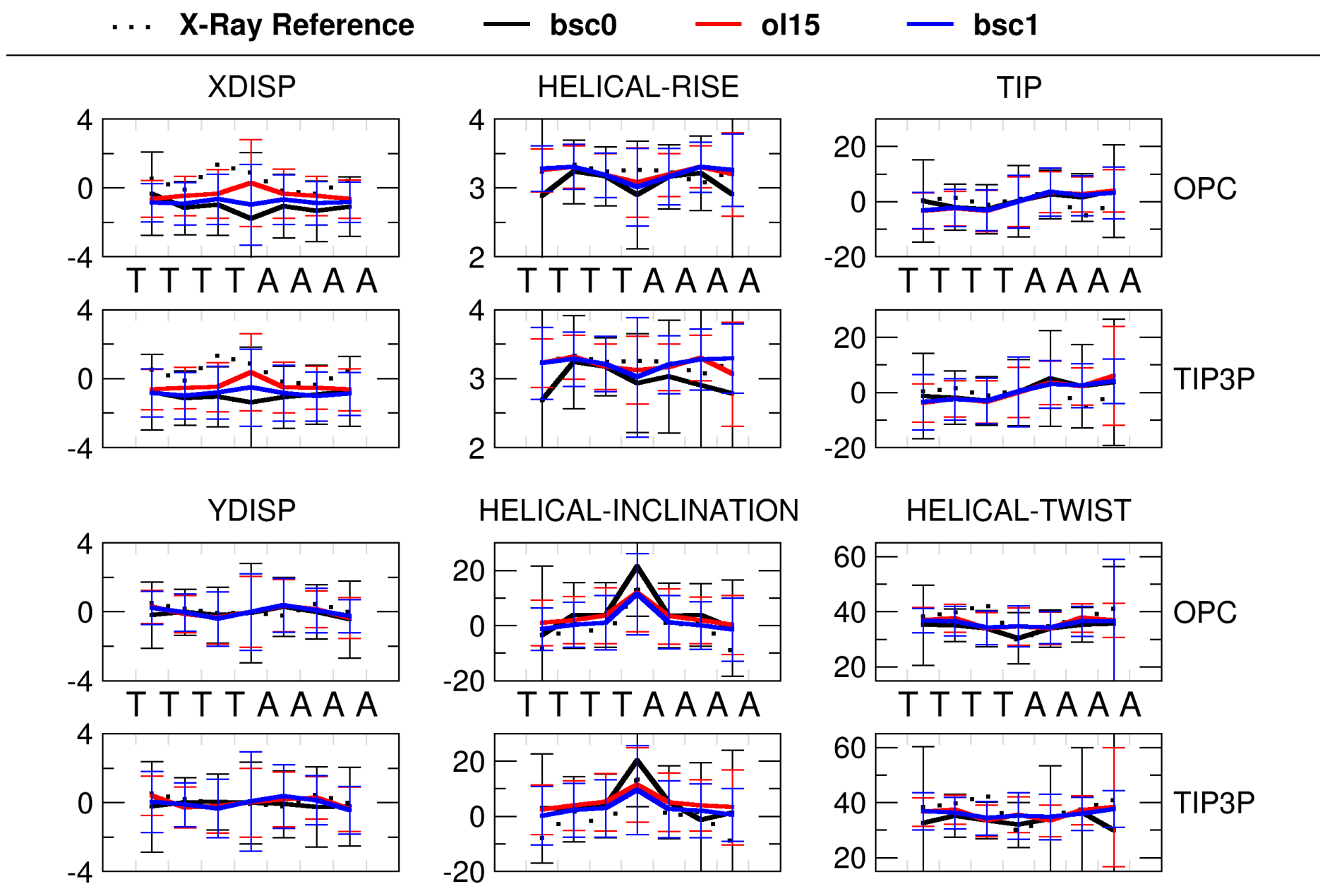


Figure S12. Selected intra-base helical parameters for individual base pairs steps in the 1 SK5 system

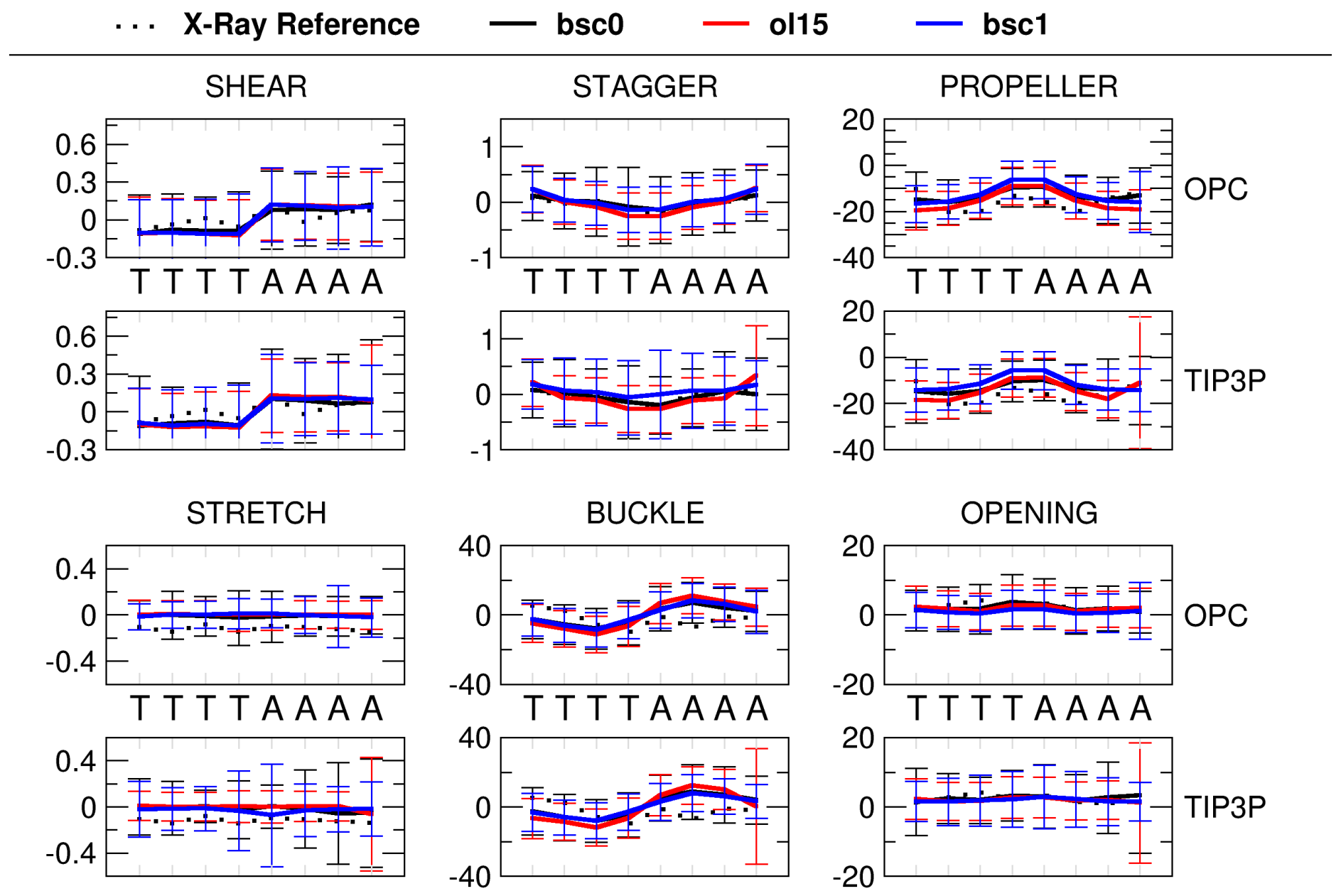


Figure S13. Selected inter-base helical parameters for individual base pairs steps in the 1 SK5 system.

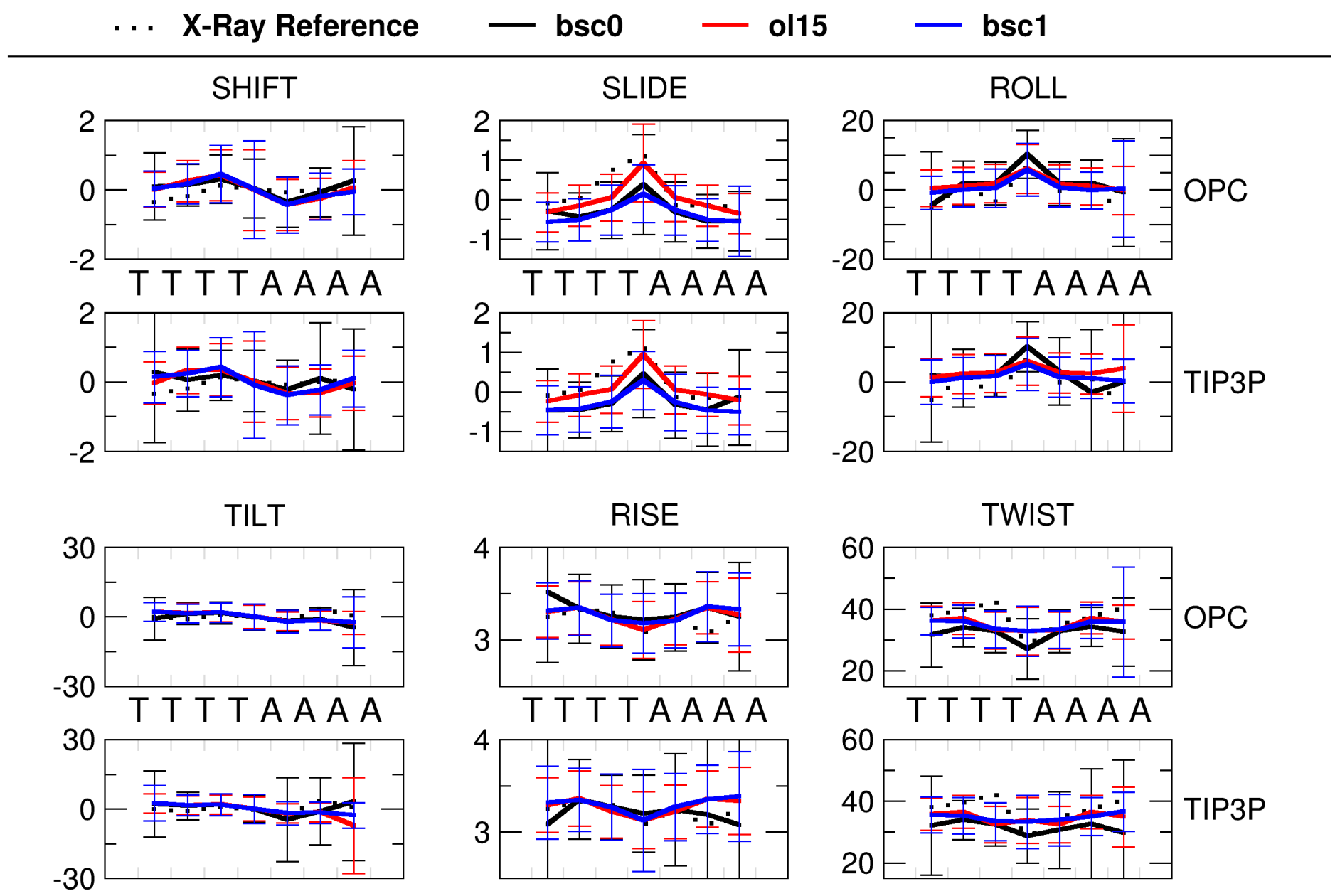


Figure S14. Selected groove widths for the 1SK5.

.. X-Ray Reference - bsc0 - ol15 - bsc1

MAJOR GROOVE WIDTH

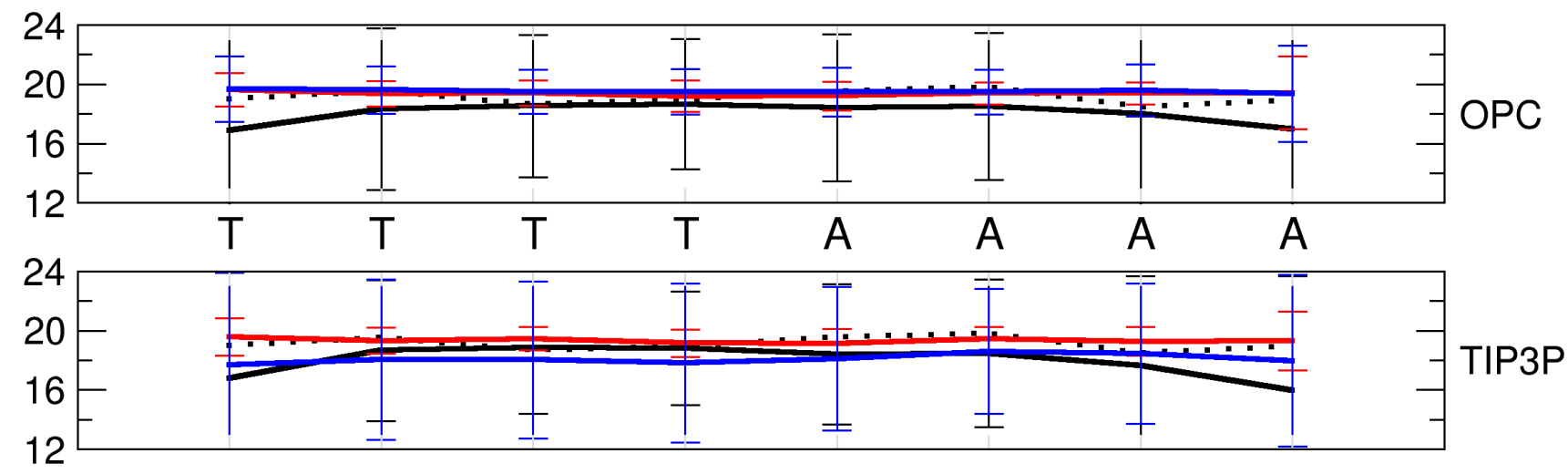

MINOR GROOVE WIDTH

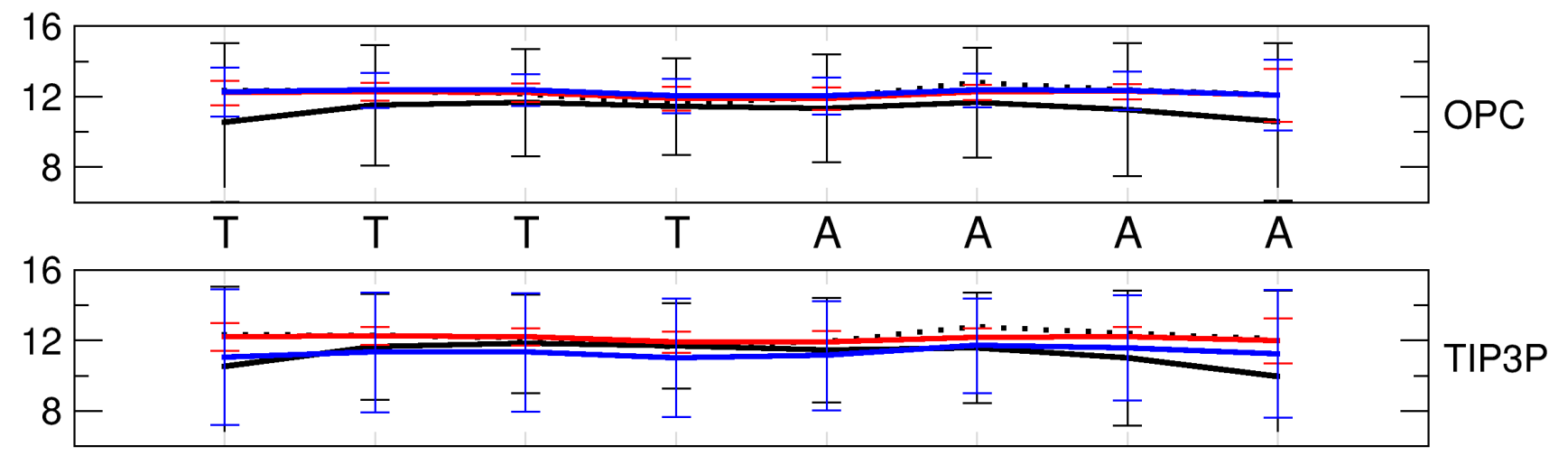


Figure S15. Selected global helical parameters for individual base pairs steps in the 3GGI system.

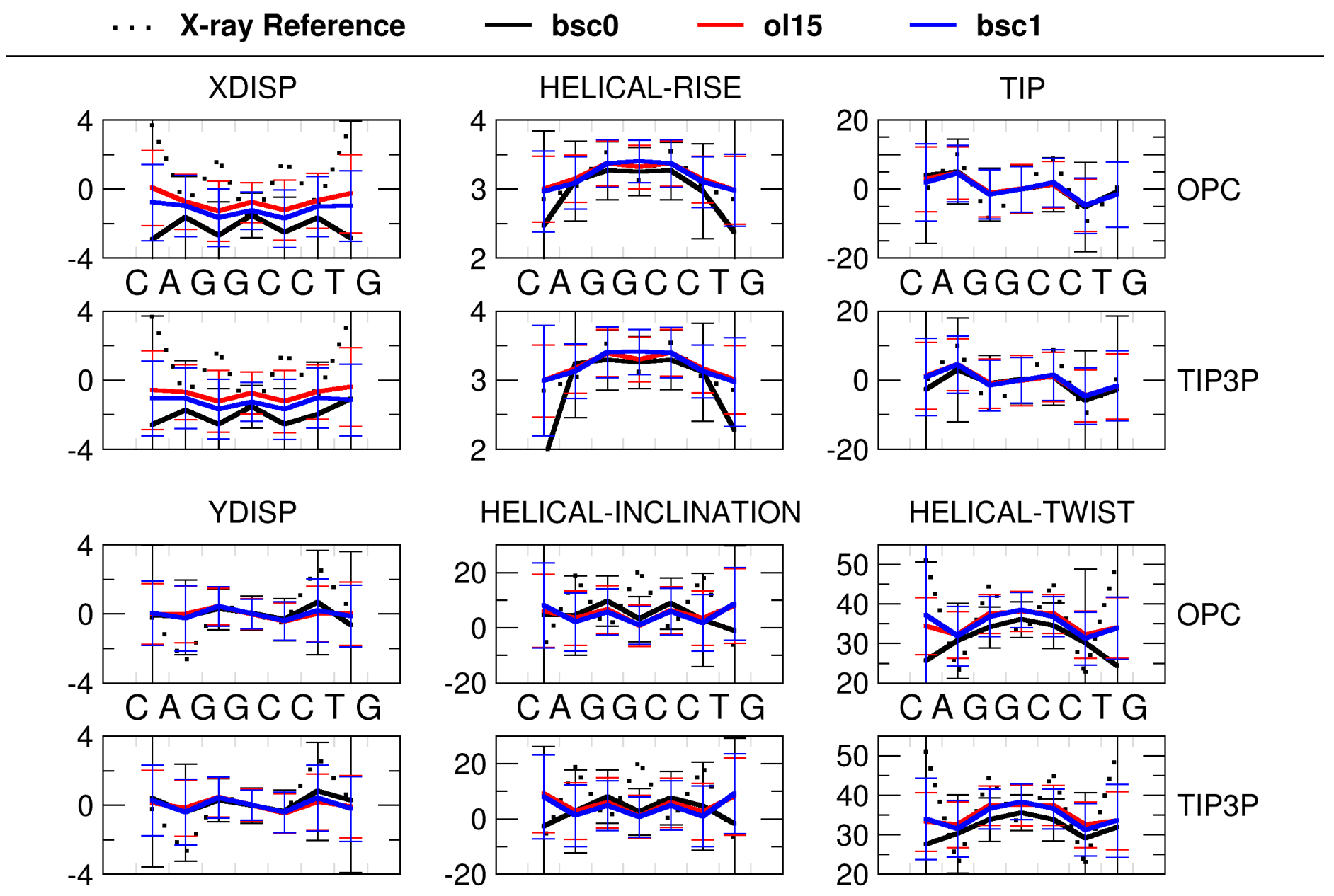


Figure $\mathbf{S} 16$ Selected intra-base helical parameters for individual base pairs steps in the 3GGI system

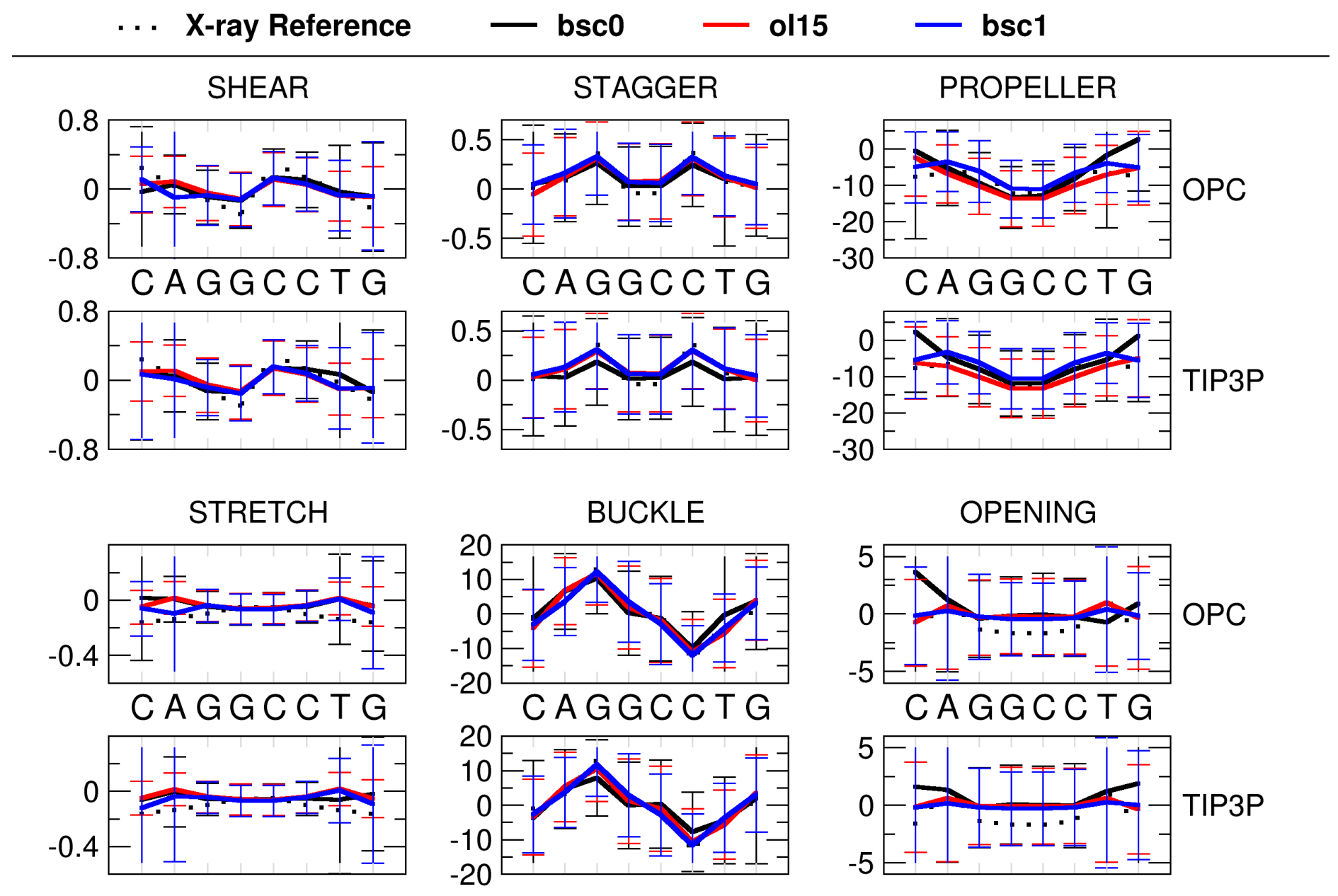


Figure S17. Selected inter-base helical parameters for individual base pairs steps in the 3GGI system.

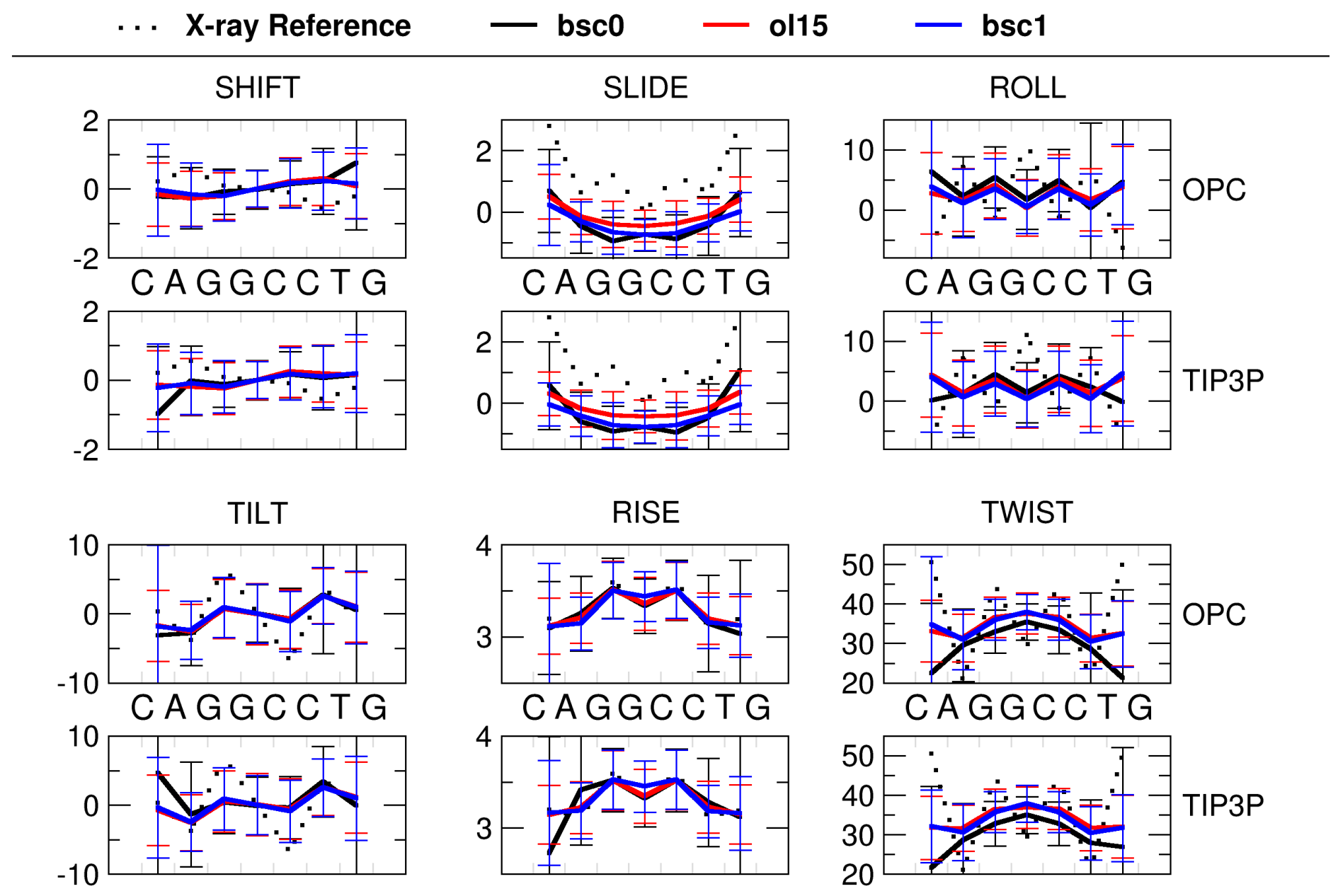


Figure S18. Selected groove widths for the 3GGI.

‥ X-ray Reference - bsc0 - ol15 - bsc1

MAJOR GROOVE WIDTH
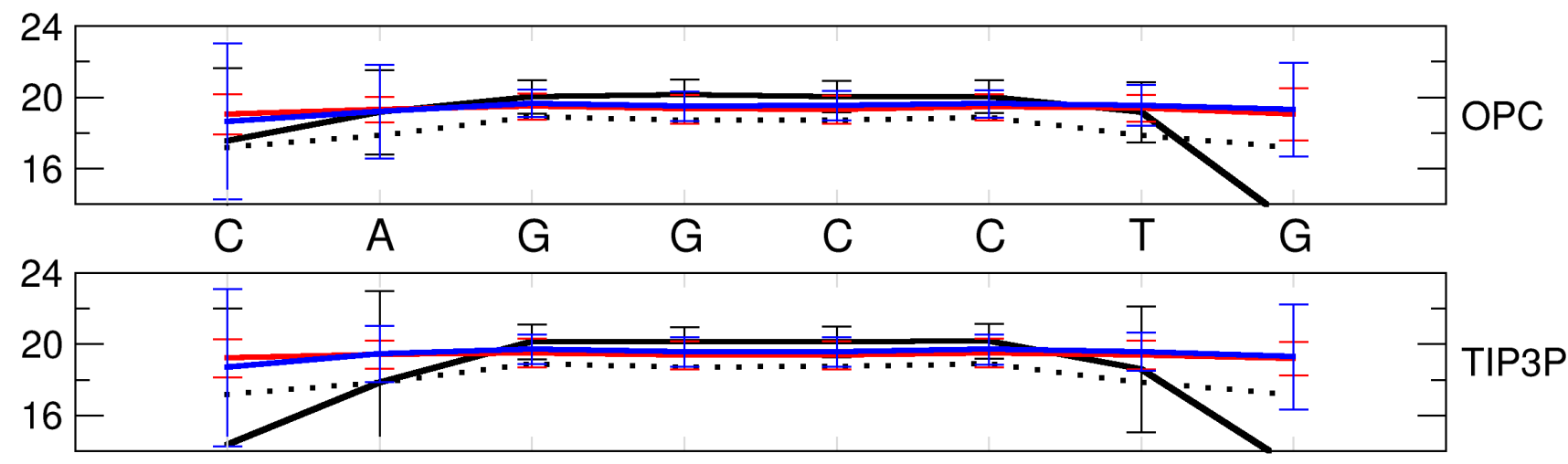

MINOR GROOVE WIDTH

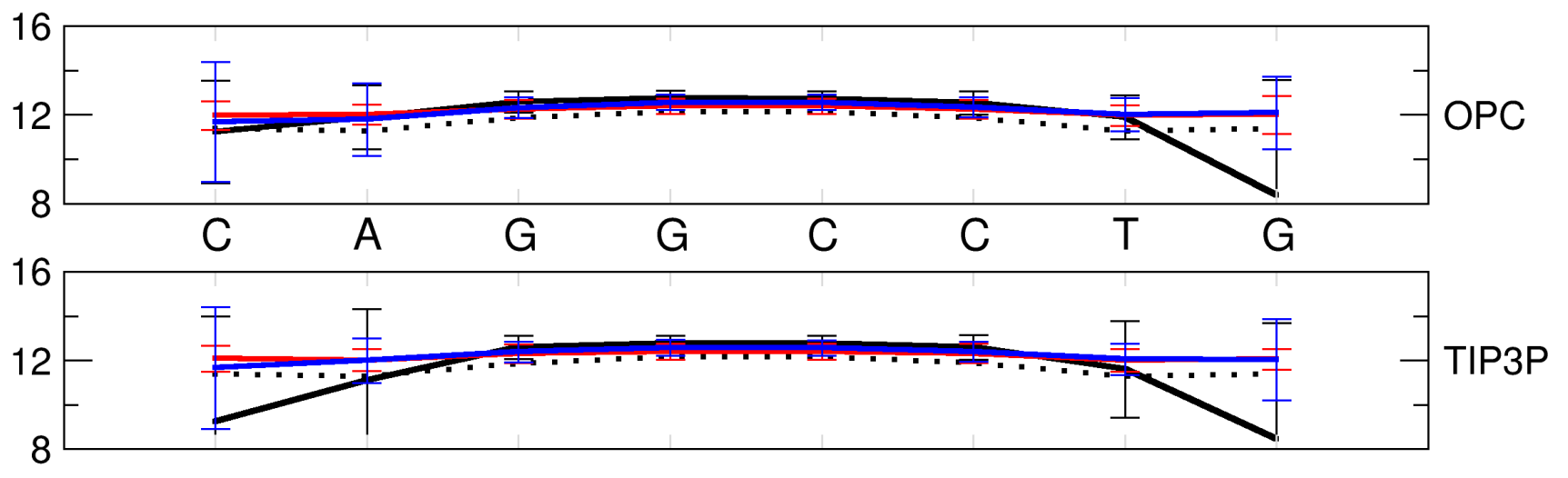


Figure S19. Selected global helical parameters for individual base pairs steps in the 110T system.

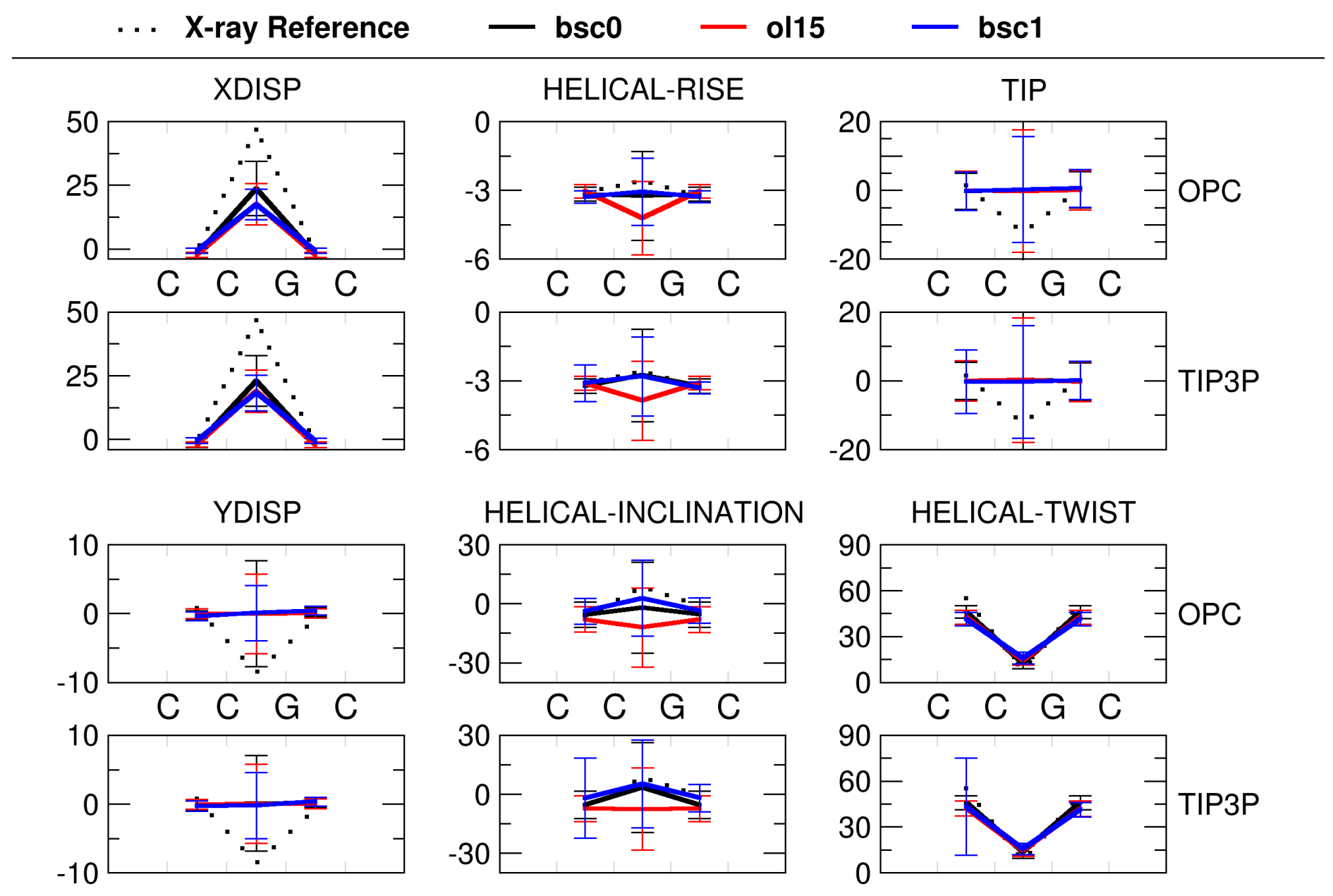


Figure S20 Selected intra-base helical parameters for individual base pairs steps in the 110T system

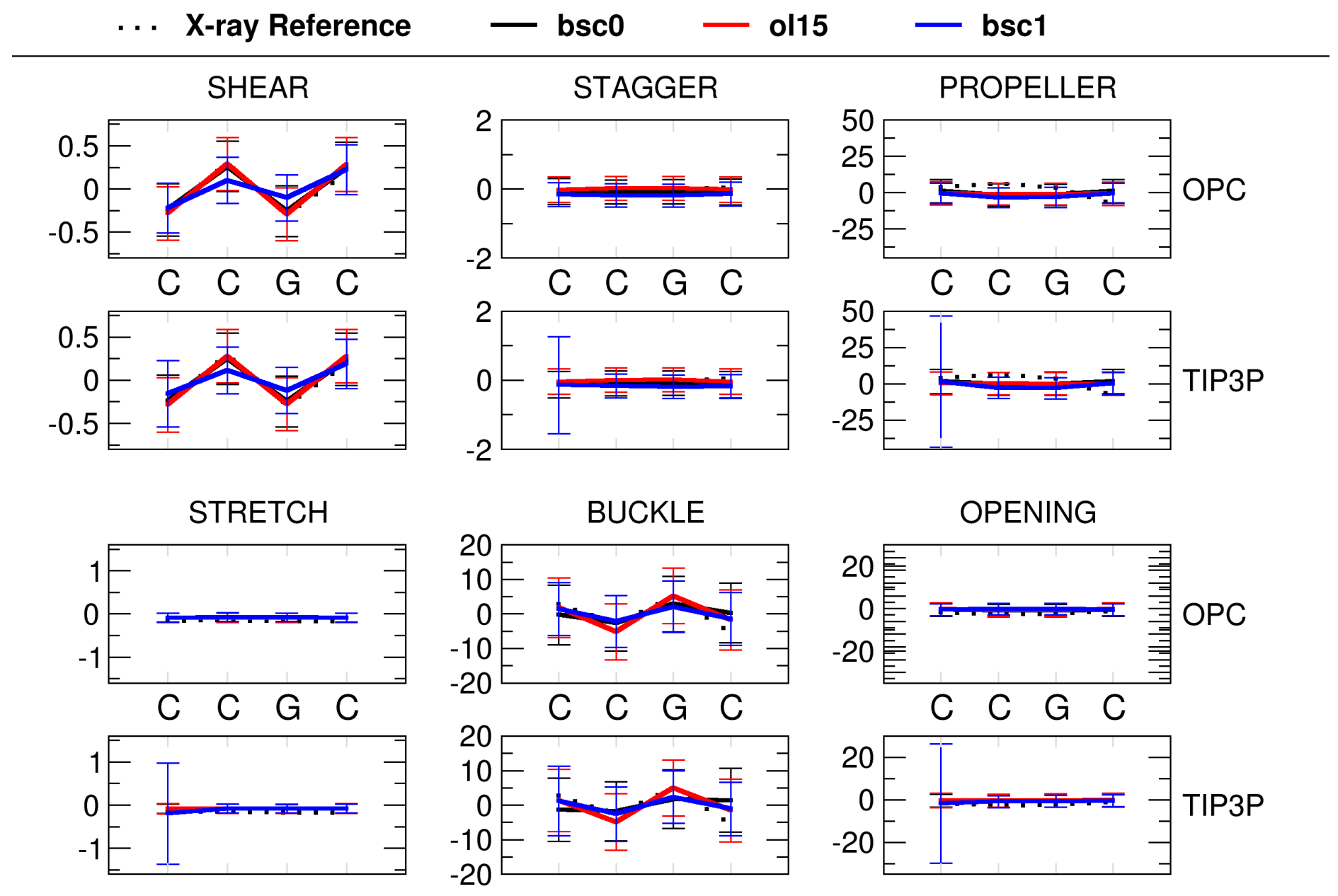


Figure S21. Selected inter-base helical parameters for individual base pairs steps in the 1I0T system.

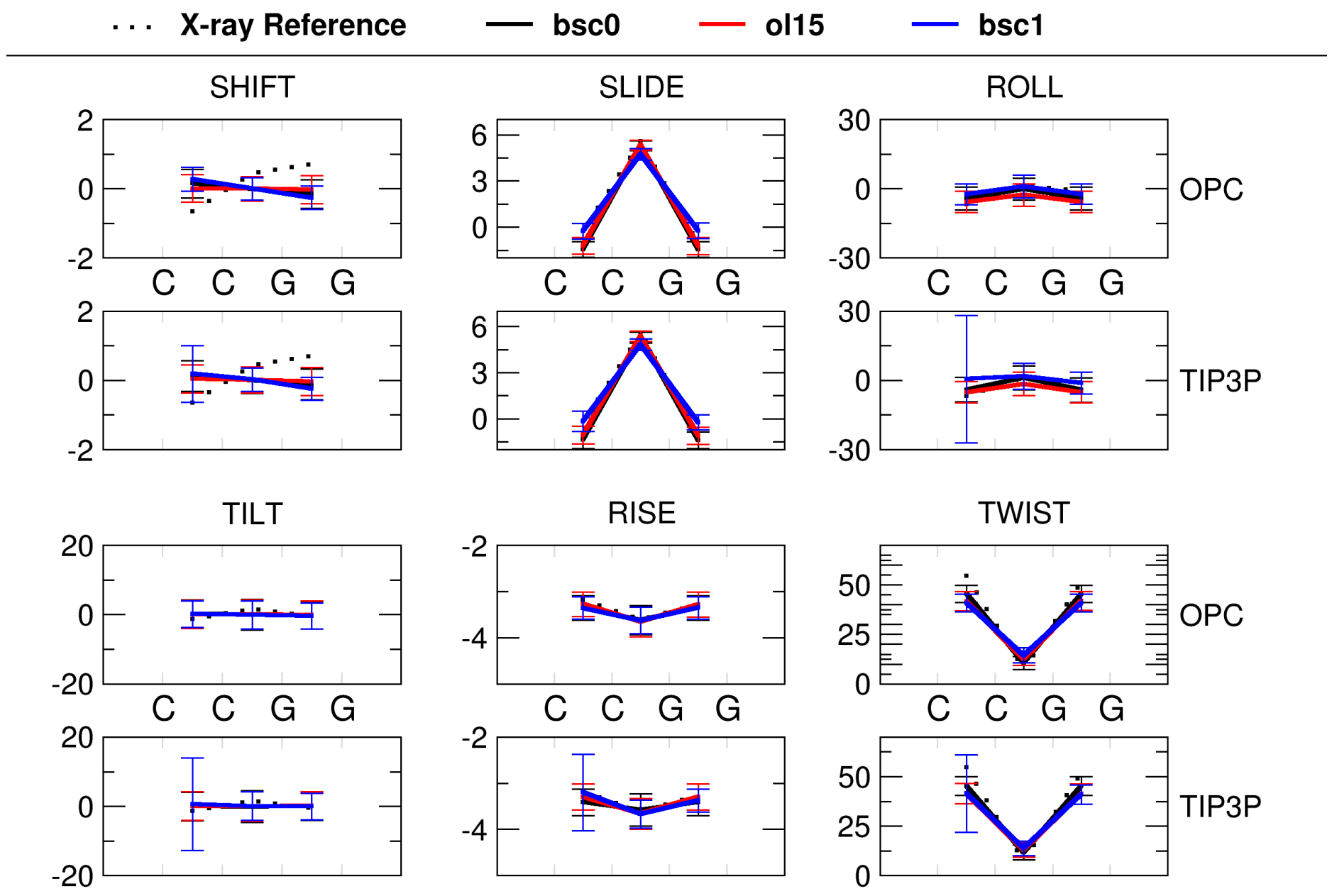


Figure S22. Selected groove widths for the 110T.

‥ X-ray Reference - bsc0 - ol15 - bsc1

MAJOR GROOVE WIDTH
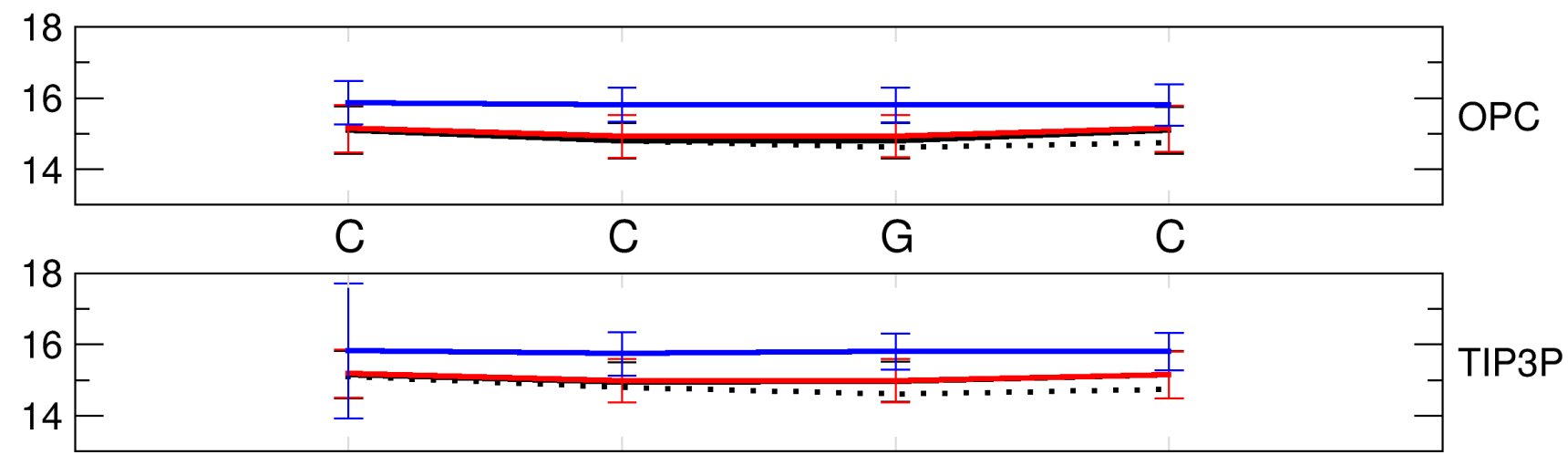

MINOR GROOVE WIDTH

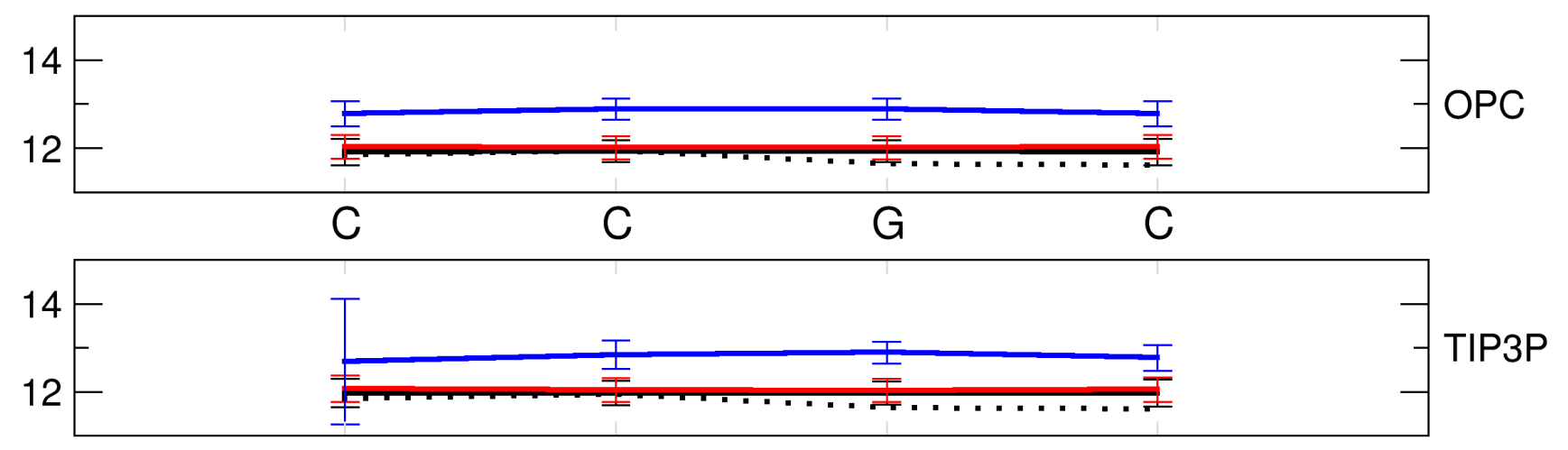

